\title{
Hierarchical structure of physical Yukawa couplings from matter field Kähler metric
}

\author{
Keiya Ishiguro, ${ }^{a}$ Tatsuo Kobayashi $^{b}$ and Hajime Otsuka ${ }^{c}$ \\ ${ }^{a}$ Department of Physics, Waseda University, \\ Tokyo 169-8555, Japan \\ ${ }^{b}$ Department of Physics, Hokkaido University, \\ Sapporo 060-0810, Japan \\ ${ }^{c}$ KEK Theory Center, Institute of Particle and Nuclear Studies, KEK, \\ 1-1 Oho, Tsukuba, Ibaraki 305-0801, Japan \\ E-mail: keyspire@ruri.waseda.jp, \\ kobayashi@particle.sci.hokudai.ac.jp, hotsuka@post.kek.jp
}

ABSTRACT: We study the impacts of matter field Kähler metric on physical Yukawa couplings in string compactifications. Since the Kähler metric is non-trivial in general, the kinetic mixing of matter fields opens a new avenue for realizing a hierarchical structure of physical Yukawa couplings, even when holomorphic Yukawa couplings have the trivial structure. The hierarchical Yukawa couplings are demonstrated by couplings of pure untwisted modes on toroidal orbifolds and their resolutions in the context of heterotic string theory with standard embedding. Also, we study the hierarchical couplings among untwisted and twisted modes on resolved orbifolds.

KEYwords: Superstrings and Heterotic Strings, Compactification and String Models

ArXiv EPrint: 2103.10240 


\section{Contents}

1 Introduction 1

2 Heterotic string theory with standard embedding 3

2.1 Effective action 3

2.2 Role of Kähler metric on physical Yukawa couplings 5

2.2.1 Calabi-Yau threefolds 5

$\begin{array}{lll}2.2 .2 & \text { Toroidal orbifolds } & 7\end{array}$

3 Hierarchical structure of physical Yukawa couplings $\quad 10$

$\begin{array}{lll}3.1 & T^{6} / \mathbb{Z}_{3} \text { orbifold } & 10\end{array}$

$\begin{array}{lll}3.2 & \text { Blown-up } T^{6} /\left(\mathbb{Z}_{2} \times \mathbb{Z}_{2}\right) \text { orbifold } & 14\end{array}$

$\begin{array}{lll}3.2 .1 & \text { Yukawa couplings of untwisted modes } & 15\end{array}$

$\begin{array}{ll}\text { 3.2.2 Yukawa couplings of the twisted mode } & 16\end{array}$

$\begin{array}{lll}3.3 & \text { A mirror dual of } T^{6} /\left(\mathbb{Z}_{3} \times \mathbb{Z}_{3}\right) \text { orbifold } & 19\end{array}$

4 Conclusions $\quad 20$

\section{Introduction}

The origin of flavor structure of quarks and leptons, in particular the hierarchical structure of their masses is one of unsolved issues in the Standard Model (SM) of particle physics. The Yukawa couplings of quarks and leptons are free parameters such that their masses and mixing angles are fitted with experimental data. There is no guiding principle to choose the Yukawa couplings in the quark sector, which have hierarchical ratios of $\mathcal{O}\left(10^{5}\right)$. Several mechanisms are proposed to explain the structure of Yukawa couplings, as represented by the localization of matter wavefunctions in the extra-dimensional space $[1,2]$ and a proper charge assignment of quarks/leptons under the continuous or discrete flavor symmetries [35]. These mechanisms can be engineered in the ultra-violet completion of the SM, such as its supersymmetric extension and the string theory.

In the four-dimensional (4D) $\mathcal{N}=1$ supersymmetric theory, physical Yukawa couplings $Y_{\hat{a} \hat{b} \hat{c}}$ are obtained from holomorphic Yukawa couplings $\kappa_{a b c}$ in the canonical basis, where a kinetic term of matter fields is canonical, i.e., the diagonal Kähler metric. Note that the Kähler metric is in a non-diagonal, but non-trivial form in a generic supergravity theory derived from the superstring theory. There are two possibilities to realize the hierarchical structure of physical Yukawa couplings: (i) the flavor structure of holomorphic Yukawa couplings controlled by the continuous/discrete flavor symmetries, (ii) a non-trivial structure of matter field Kähler metric which would be connected with the geometry of extra-dimensional space. 
In the traditional approach (i), holomorphic Yukawa couplings are well controlled by stringy selection rules, gauge and extra-dimensional geometrical symmetries. It was known that the holomorphic Yukawa couplings of twisted modes on heterotic orbifold models are exponentially suppressed with respect to moduli fields [6-8]. Hence, one can realize the hierarchical structure of physical Yukawa couplings of these states due to the fact that they are localized at fixed points. However, the coupling selection rules among twisted modes are very tight $[9,10]$. It is not straightforward to realize realistic mass matrices for three generations of quarks and leptons. (See for attempts to realize quark and lepton mass matrices by twisted modes, refs. [11, 12].)

At any rate, it would be difficult to have the hierarchical structure for other states such as untwisted modes propagating the bulk. It indicates that smooth Calabi-Yau (CY) compactifications will not lead to the hierarchical structure of holomorphic Yukawa couplings for matter zero-modes. For instance, in the context of heterotic string theory with standard embedding, the holomorphic Yukawa couplings are of $\mathcal{O}(1)$ in the large volume/complex structure regime of CY threefolds, determined by the third derivative of the prepotential. It results in the trivial structure of the holomorphic Yukawa couplings which are also observed in the untwisted modes on a singular limit of CY threefolds such as toroidal orbifolds. Hence, the understanding of matter field Kähler metric would shed new light on the structure of physical Yukawa couplings.

In this paper, we study whether the matter field Kähler metric provides the hierarchical structure of physical Yukawa couplings. In contrast to the holomorphic Yukawa couplings, the matter field Kähler metric would not be restricted by symmetry arguments. Hence, in the approach (ii), we have to calculate zero-mode wavefunctions themselves living on the extra-dimensional space to understand the structure of matter field Kähler metric. ${ }^{1}$ To overcome this problem, we focus on the heterotic string theory with standard embedding, where matter fields are in one-to-one correspondence with moduli fields on CY threefolds including toroidal orbifolds in the singular limit. In this context, the matter field Kähler metric as well as the holomorphic Yukawa couplings are explicitly calculated by means of conformal field theory as well as the special geometry [18]. To study the impacts of matter field Kähler metric on physical Yukawa couplings, we deal with a simplified CY threefold and toroidal orbifolds preserving the supersymmetry. On toroidal backgrounds, the moduli fields are categorized by untwisted and twisted sectors, where twisted modes are analyzed through resolutions of toroidal orbifolds, capturing the feature of CY threefolds. We find that the kinetic mixings on the moduli space of untwisted and twisted modes are inevitable for a realization of hierarchical Yukawa couplings. Remarkably, $\mathcal{O}(1)$ values of the moduli fields provide the hierarchical structure of physical Yukawa couplings, demonstrated on specific toroidal orbifolds with and without resolutions having the non-diagonal matter field Kähler metric.

The remaining part of this paper is organized as follows. In section 2, we briefly review an effective action of matter fields, focusing on Yukawa couplings for the fundamental

\footnotetext{
${ }^{1}$ Explicit calculations of zero-mode wavefunctions were performed on e.g., toroidal backgrounds with line bundles [13-15] and local 4-cycle on the conifold regime of CY threefold [16] in Type IIB string theory and smooth CY threefolds with large internal gauge fluxes [17] in the context of heterotic string theory.
} 
and anti-fundamental representations of $E_{6}$ gauge group. The off-diagonal entries in the matter field Kähler metric play an important role of distinguishing the holomorphic Yukawa couplings and the physical one as illustrated on toroidal orbifolds in detail. In section 3 , the hierarchical physical Yukawa couplings are demonstrated on $T^{6} / \mathbb{Z}_{3}$ orbifold, blown-up $T^{6} /\left(\mathbb{Z}_{2} \times \mathbb{Z}_{2}\right)$ orbifold and a mirror dual of $T^{6} /\left(\mathbb{Z}_{3} \times \mathbb{Z}_{3}\right)$ orbifold. Finally, section 4 is devoted to the conclusion and discussion.

\section{$2 \quad$ Heterotic string theory with standard embedding}

In section 2.1, we briefly review the effective action of matter fields in the heterotic string theory on CY compactification with standard embedding. In particular, we focus on Yukawa couplings of matter zero-modes, determined by triple intersection numbers of CY threefolds. In section 2.2, the matter kinetic mixings on a simplified CY threefold and toroidal orbifolds are examined in the light of physical Yukawa couplings.

\subsection{Effective action}

The effective action of matter fields is well developed in the context of heterotic string theory on CY threefolds with standard embedding, where zero-modes are characterized by a cohomology basis of $\mathrm{CY}$ threefolds. ${ }^{2}$ From the identification of $\mathrm{SU}(3) \subset E_{8}$ gauge bundle with a tangent bundle of $\mathrm{CY}$ threefolds $\mathcal{M}, E_{8}$ gauge group is broken to $E_{6}$ one. Correspondingly, the fundamental and anti-fundamental representations of $E_{6}$ are spanned by the $H^{1,1}(\mathcal{M})$ and $H^{2,1}(\mathcal{M})$ cohomology bases, respectively.

We begin with the effective action of moduli fields, whose Kähler potential is described by

$$
K=K_{\mathrm{ks}}(T, \bar{T})+K_{\mathrm{cs}}(U, \bar{U}),
$$

where $K_{\mathrm{ks}}(T, \bar{T})$ and $K_{\mathrm{cs}}(U, \bar{U})$ denote the Kähler potential of the Kähler moduli $T^{a}$ and the complex structure moduli $U^{i}$, respectively. It is known that they are described by a complexified Kähler form $J$ and a holomorphic three-form $\Omega$ of CY threefolds:

$$
\begin{aligned}
& K_{\mathrm{ks}}=-\ln \left(\int_{\mathcal{M}} J \wedge J \wedge J\right), \\
& K_{\mathrm{cs}}=-\ln \left(-i \int_{\mathcal{M}} \Omega \wedge \Omega\right) .
\end{aligned}
$$

In the large volume and complex structure regime of $\mathrm{CY}$ threefolds, we can expand the complexified Kähler form and the holomorphic three-form on the basis of $H^{1,1}(\mathcal{M})$ and $H^{2,1}(\mathcal{M})$ :

$$
J=\sum_{a=1}^{h^{1,1}}\left(t^{a}+i b^{a}\right) w_{a}, \quad \Omega=\sum_{I=0}^{h^{2,1}}\left(X^{I} \alpha_{I}-F_{I} \beta^{I}\right),
$$

from which the Kähler moduli are defined as $T^{a} \equiv t^{a}+i b^{a}$ on the basis of Kähler form $w_{a}$ with $a$ running over the number of two-cycles of CY threefolds, namely the hodge number

\footnotetext{
${ }^{2}$ For more detailed discussions, we refer to e.g., refs. [19, 20].
} 
$h^{1,1}$. Here, $t^{a}$ and $b^{a}$ denote the volume of two-cycle and Kähler axions originating from Kalb-Ramond two-form $B^{(2)}$, respectively.

On the other hand, the holomorphic three-form $\Omega$ is expanded on the canonical symplectic basis $\left\{\alpha_{I}, \beta^{I}\right\}$ satisfying

$$
\int_{\mathcal{M}} \alpha_{I} \wedge \beta^{I}=\delta_{I}^{J}, \quad \int_{\mathcal{M}} \alpha_{I} \wedge \alpha_{J}=\int_{\mathcal{M}} \beta^{I} \wedge \beta^{J}=0,
$$

on which the coefficient $F_{I}=\partial_{I} F$ corresponds to the first derivative of the prepotential $F$ with respect to $X^{I}$. In what follows, we adopt the gauge $X^{0}=1$, yielding the complex structure moduli in the flat coordinate; $X^{i}=U^{i},\left(i=1,2, \cdots, h^{2,1}\right)$ with $h^{2,1}$ being the hodge number of $\mathrm{CY}$ threefolds. There the complex structure moduli space is governed by the structure of the prepotential whose functional form is known in the large complex structure regime,

$$
F(U)=\frac{1}{3 !} \kappa_{i j k} U^{i} U^{j} U^{k}
$$

We now pick up the cubic term determined by the triple intersection numbers $\kappa_{i j k}$, but other next-leading terms are irrelevant to the following discussions. Furthermore, it is possible to consider the other corner of complex structure moduli space, but the prepotential has a model-dependent structure as shown in an example of section 3.3. Then, the Kähler potential (2.2) is simplified as follows:

$$
\begin{aligned}
& K_{\mathrm{cs}}=-\ln \left[\frac{i}{6} \kappa_{i j k}\left(U^{i}-\bar{U}^{i}\right)\left(U^{j}-\bar{U}^{j}\right)\left(U^{k}-\bar{U}^{k}\right)\right], \\
& K_{\mathrm{ks}}=-\ln \left[\frac{1}{6} \kappa_{a b c}\left(T^{a}+\bar{T}^{a}\right)\left(T^{b}+\bar{T}^{b}\right)\left(T^{c}+\bar{T}^{c}\right)\right],
\end{aligned}
$$

where it is notable that loop and quantum corrections are assumed to be enough suppressed in comparison with the classical terms.

We next deal with the effective action of charged matter fields 27 and $\overline{27}$ under the $E_{6}$ gauge group, which are denoted by $A^{a}$ and $A^{i}$, respectively. The matter Kähler potential is only known in an expansion of $A^{a}$ and $A^{i}$, that is, $A^{a}, A^{i} \ll 1[18]$, and the corresponding Kähler metrics become

$$
\begin{aligned}
& K_{a \bar{b}}^{(27)}=e^{\frac{1}{3}\left(K_{\mathrm{cs}}-K_{\mathrm{ks}}\right)}\left(K_{\mathrm{ks}}\right)_{a \bar{b}} \\
& K_{i \bar{j}}^{(2 \overline{2})}=e^{-\frac{1}{3}\left(K_{\mathrm{cs}}-K_{\mathrm{ks}}\right)}\left(K_{\mathrm{cs}}\right)_{i \bar{j}} .
\end{aligned}
$$

The matter field Kähler metric is in turn in one-to-one correspondence with the moduli Kähler metric. In addition, Yukawa interactions of charged matter fields are described by the following superpotential:

$$
W=\kappa_{a b c} A^{a} A^{b} A^{c}+\kappa_{i j k} A^{i} A^{j} A^{k}
$$

stating that the holomorphic $27^{3}$ and $\overline{27}^{3}$ Yukawa couplings are determined by the triple intersection numbers of the Kähler and complex structure moduli, respectively [21, 22]. To 
derive the physical Yukawa couplings, we perform the unitary transformation $L$ diagonalizing the kinetic terms for multiple 27 matter fields, ${ }^{3}$

$$
A^{a} K_{a \bar{b}}^{(27)} \bar{A}^{\bar{b}}=e^{\frac{1}{3}\left(K_{\mathrm{cs}}-K_{\mathrm{ks}}\right)} A^{a}\left(L^{\dagger}\right)_{a}^{\overline{\hat{c}}} \Lambda_{\overline{\hat{c}} \hat{c}} L_{\bar{b}}^{\hat{c}} \bar{A}^{\bar{b}} \equiv\left|\mathcal{A}^{\hat{a}}\right|^{2},
$$

where $\Lambda$ stands for an eigenvalue matrix satisfying $L K L^{\dagger}=\Lambda$. The canonically normalized matter fields $\mathcal{A}^{\hat{a}}$ are defined as

$$
\mathcal{A}^{\hat{a}}=e^{\frac{1}{6}\left(K_{\mathrm{cs}}-K_{\mathrm{ks}}\right)} A^{c}\left(L^{\dagger}\right)_{c}^{\hat{a}}(\sqrt{\Lambda})_{\hat{a} \overline{\hat{a}}}=e^{\frac{1}{6}\left(K_{\mathrm{cs}}-K_{\mathrm{ks}}\right)} A^{c}\left(L^{\dagger} \sqrt{\Lambda}\right)_{c}^{\hat{a}}
$$

and the physical Yukawa couplings result in

$$
e^{K / 2} \kappa_{d e f} A^{d} A^{e} A^{f}=e^{K_{\mathrm{ks}}} \kappa_{d e f} \mathcal{A}^{\hat{a}} \mathcal{A}^{\hat{b}} \mathcal{A}^{\hat{c}}\left(\Lambda^{-1 / 2} L\right)_{\hat{a}}^{d}\left(\Lambda^{-1 / 2} L\right)_{\hat{b}}^{e}\left(\Lambda^{-1 / 2} L\right)_{\hat{c}}^{f} \equiv Y_{\hat{a} \hat{b} \hat{c}} \mathcal{A}^{\hat{a}} \mathcal{A}^{\hat{b}} \mathcal{A}^{\hat{c}},
$$

with $^{4}$

$$
Y_{\hat{a} \hat{b} \hat{c}} \equiv e^{K_{\mathrm{ks}}}\left(\Lambda^{-1 / 2} L\right)_{\hat{a}}^{d}\left(\Lambda^{-1 / 2} L\right)_{\hat{b}}^{e}\left(\Lambda^{-1 / 2} L\right)_{\hat{c}}^{f} \kappa_{d e f} .
$$

Note that not all the 27 and $\overline{27}$ representations behave as the chiral zero-modes in the low-energy effective action. The index theorem tells us that the net number of chiral zero-modes is determined by the index

$$
\frac{\chi(\mathcal{M})}{2}=h^{1,1}-h^{2,1}
$$

corresponding to half the Euler characteristics of CY threefolds $\chi(\mathcal{M})$. Hence, the chiral zero-modes in our interest are the remaining zero-modes originating from 27 or $\overline{27}$. Remarkably, the holomorphic Yukawa couplings in both 27 and $\overline{27}$ sectors are integers determined by the triple intersection numbers, indicating that it would be difficult to realize the hierarchical structure of holomorphic Yukawa couplings. Indeed, the difficulties of hierarchical structure of holomorphic Yukawa couplings on CY threefolds and toroidal orbifolds are discussed in detail in the following sections.

\subsection{Role of Kähler metric on physical Yukawa couplings}

In this section, we will focus on kinetic mixings to exemplify the difference between the physical Yukawa couplings and the holomorphic one on the basis of a simplified CY threefold in section 2.2.1 and toroidal orbifolds in section 2.2.2.

\subsubsection{Calabi-Yau threefolds}

We start with CY threefolds to show the impacts of Kähler mixing on physical Yukawa couplings. It was known that typical CY threefolds contain hodge numbers with $\mid h^{1,1}-$ $h^{2,1} \mid=\mathcal{O}(100)$ as shown in Kreuzer-Skarke dataset of CY threefolds in toric ambient

\footnotetext{
${ }^{3}$ Here, we focus on the 27 sector, but it is straightforward to extend the following calculation to $\overline{27}$ sector. Furthermore, we note that the hat index is introduced to canonically normalized fields in subsequent analyses.

${ }^{4}$ Physical $\overline{27}^{3}$ Yukawa couplings have an overall factor $e^{K_{\mathrm{cs}}}$, whose factor is important to obtain the gauge invariant quantity under the symplectic group.
} 
spaces [23, 24], leading to $\mathcal{O}(100)$ number of chiral zero-modes. (See for several attempts to construct CY threefolds with a small hodge number, e.g., ref. [25].)

For the illustrative purpose, we assume that CY threefolds have the hodge number $h^{1,1}-h^{2,1}=3$ and the moduli space of remaining three-generation zero-modes is described by the special Kähler manifold $\left(\frac{\mathrm{SU}(1,1)}{\mathrm{U}(1)}\right)^{3}$. The Kähler potential of this geometry is described by

$$
K=-\sum_{a=1}^{3} \ln \left(T^{a}+\bar{T}^{a}\right),
$$

where $T^{a}$ denotes the Kähler moduli protected from becoming massive. The triple intersection number of these moduli is found as

$$
\kappa_{123}=1
$$

and 0 otherwise, and these correspond to the holomorphic $27^{3}$ Yukawa couplings. For concreteness, we identify the Higgs field with an element of $A^{1}$ and suppose that three generations of quarks and leptons appear from $A^{a}(a=1,2,3)$, associated with the massless Kähler moduli. Then, the holomorphic Yukawa couplings of quarks and leptons on the $\left(A^{1}, A^{2}, A^{3}\right)$ basis are of the form:

$$
\kappa_{1 a b}=\left(\begin{array}{lll}
0 & 0 & 0 \\
0 & 0 & 1 \\
0 & 1 & 0
\end{array}\right) .
$$

This rank-two matrix does not have non-trivial flavor and hierarchical structures. On these backgrounds, the Kähler metric is of the diagonal form due to the factorizable Kähler metric:

$$
K_{a \bar{b}}=\frac{\delta_{a \bar{b}}}{\left(T^{a}+\bar{T}^{a}\right)^{2}},
$$

yielding the non-vanishing element of physical Yukawa matrix

$$
Y_{\hat{1} \hat{2} \hat{3}}=e^{K_{\mathrm{ks}}}\left(\Lambda^{-1 / 2} L\right)_{\hat{1}}^{1}\left(\Lambda^{-1 / 2} L\right)_{\hat{2}}^{2}\left(\Lambda^{-1 / 2} L\right)_{\hat{3}}^{3} \kappa_{123},
$$

with the eigenvalue matrix $\Lambda$ and the diagonalizing matrix $L$ of moduli Kähler metric. Since the physical Yukawa couplings have the same structure as the holomorphic Yukawa couplings, it is still difficult to realize the non-trivial flavor and hierarchical structure of Yukawa couplings on such a special Kähler manifold with $\left(\frac{\mathrm{SU}(1,1)}{\mathrm{U}(1)}\right)^{3}$.

When the Kähler moduli space differs from the $\left(\frac{\mathrm{SU}(1,1)}{\mathrm{U}(1)}\right)^{3}$ structure, a generic Kähler potential for the remaining 3 massless modes would be given by

$$
K=-\ln \left[\kappa_{a b c}\left(T^{a}+\bar{T}^{a}\right)\left(T^{b}+\bar{T}^{b}\right)\left(T^{c}+\bar{T}^{c}\right)+\cdots\right],
$$

where the ellipsis represents contributions from other massive moduli. The matter kinetic mixings are thus induced by the intersection number $\kappa_{a b c}$ and/or vacuum expectation values of heavy moduli, leading to the non-trivial flavor structure of 27 zero-modes. The 
structure of physical Yukawa couplings (2.12) would be different from the holomorphic one due to the matter Kähler mixings. So far, we have focused on the Kähler moduli sector accompanying the 27 zero-modes, but these statements hold for the opposite case with $h^{2,1}>h^{1,1}$, where $\overline{27}$ zero-modes are in one-to-one correspondence with the complex structure moduli. Note that the holomorphic $\overline{27}^{3}$ Yukawa couplings are also determined by triple intersection numbers $\kappa_{i j k}$ in the moduli space with the large complex structure.

However, there is no definite way to analyze the CY moduli space with a large Euler number, although there exist the early three-generation models on a specific CY threefold taking into account freely acting discrete symmetries $[26,27]$. In this respect, we move on to toroidal orbifolds with and without resolutions, on which physical Yukawa couplings of untwisted and twisted modes are analyzed in detail below. The blown-up toroidal orbifolds would capture the structure of physical Yukawa couplings on smooth CY threefolds.

\subsubsection{Toroidal orbifolds}

The supersymmetric orbifold backgrounds are classified in refs. [28-30], which are summarized in table 1 for $T^{6} / \mathbb{Z}_{N}$ and $T^{6} /\left(\mathbb{Z}_{N} \times \mathbb{Z}_{M}\right)$ orbifolds. (See for Lie lattices of $T^{6}$ refs. $[9,31]$ and for further classifications of orbifolds including non-Abelian ones, e.g., ref. [32].)

The Kähler potential of the untwisted moduli fields are categorized as follows [33-35]:

1. $T^{6} / \mathbb{Z}_{3}$ orbifold

$$
K=-\ln \operatorname{det}\left(T^{a \bar{b}}+\bar{T}^{a \bar{b}}\right), \quad(a, b=1,2,3) .
$$

2. $T^{6} / \mathbb{Z}_{4,6}$ orbifolds

$$
K=-\ln \left(T^{1}+\bar{T}^{1}\right)-\ln \operatorname{det}\left(T^{a \bar{b}}+\bar{T}^{a \bar{b}}\right)-\sum_{n=1}^{h_{\text {untw. }}^{2,1}} \ln \left(U^{n}+\bar{U}^{n}\right), \quad(a, b=2,3),
$$

where the number of untwisted complex structure moduli is listed in table 1.

3. $T^{6} / \mathbb{Z}_{7,8,12}$ and $T^{6} /\left(\mathbb{Z}_{N} \times \mathbb{Z}_{M}\right)$ orbifolds

$$
K=-\sum_{a=1}^{3} \ln \left(T^{a}+\bar{T}^{a}\right)-\sum_{n=1}^{h_{\text {untw. }}^{2,1}} \ln \left(U^{n}+\bar{U}^{n}\right),
$$

where the number of untwisted complex structure moduli is listed in table 1.

The number of the Kähler moduli is larger than that of the complex structure moduli in all the toroidal orbifolds, and consequently the chiral zero-modes are originating from the Kähler moduli. ${ }^{5}$ In this respect, we examine the holomorphic $27^{3}$ Yukawa couplings.

\footnotetext{
${ }^{5}$ It is also possible to consider the opposite case, that is, $h^{2,1}>h^{1,1}$ by choosing the proper assignment of discrete torsion [30]. Indeed, our analysis is applicable to that case as demonstrated on a mirror dual of the $T^{6} /\left(\mathbb{Z}_{3} \times \mathbb{Z}_{3}\right)$ orbifold in section 3.3.
} 


\begin{tabular}{|c|c|c|c|c|c|}
\hline $\mathbb{Z}_{N}$ & Lattice & $h_{\text {untw. }}^{1,1}$ & $h_{\mathrm{twist}}^{1,1}$ & $h_{\text {untw. }}^{2,1}$ & $h_{\text {twist }}^{2,1}$ \\
\hline $\mathbb{Z}_{3}$ & $\mathrm{SU}(3)^{3}$ & 9 & 27 & 0 & 0 \\
\hline \multirow[t]{3}{*}{$\mathbb{Z}_{4}$} & $\mathrm{SU}(2)^{2} \times \mathrm{SO}(5)^{2}$ & 5 & 26 & 1 & 6 \\
\hline & $\mathrm{SU}(2) \times \mathrm{SU}(4) \times \mathrm{SO}(5)$ & 5 & 22 & 1 & 2 \\
\hline & $\mathrm{SU}(4)^{2}$ & 5 & 20 & 1 & 0 \\
\hline \multirow[t]{2}{*}{$\mathbb{Z}_{6-I}$} & $\mathrm{SU}(3) \times G_{2}^{2}$ & 5 & 24 & 0 & 5 \\
\hline & $\mathrm{SU}(3)^{2} \times G_{2}$ & 5 & 20 & 0 & 1 \\
\hline \multirow[t]{4}{*}{$\mathbb{Z}_{6-I I}$} & $\mathrm{SU}(2)^{2} \times \mathrm{SU}(3) \times G_{2}$ & 3 & 32 & 1 & 10 \\
\hline & $\mathrm{SU}(3) \times \mathrm{SO}(8)$ & 3 & 26 & 1 & 4 \\
\hline & $\mathrm{SU}(2)^{2} \times \mathrm{SU}(3) \times \mathrm{SU}(3)$ & 3 & 28 & 1 & 6 \\
\hline & $\mathrm{SU}(2) \times \mathrm{SU}(6)$ & 3 & 22 & 1 & 0 \\
\hline $\mathbb{Z}_{7}$ & $\mathrm{SU}(7)$ & 3 & 21 & 0 & 0 \\
\hline \multirow[t]{2}{*}{$\mathbb{Z}_{8-I}$} & $\mathrm{SU}(4)^{2}$ & 3 & 21 & 0 & 0 \\
\hline & $\mathrm{SO}(5) \times \mathrm{SO}(9)$ & 3 & 24 & 0 & 3 \\
\hline \multirow[t]{2}{*}{$\mathbb{Z}_{8-I I}$} & $\mathrm{SU}(2) \times \mathrm{SO}(10)$ & 3 & 24 & 1 & 2 \\
\hline & $\mathrm{SO}(4) \times \mathrm{SO}(9)$ & 3 & 28 & 1 & 6 \\
\hline \multirow[t]{2}{*}{$\mathbb{Z}_{12-I}$} & $\mathrm{SU}(3) \times F_{4}$ & 3 & 26 & 0 & 5 \\
\hline & $E_{6}$ & 3 & 22 & 0 & 1 \\
\hline $\mathbb{Z}_{12-I I}$ & $\mathrm{SO}(4) \times F_{4}$ & 3 & 28 & 1 & 6 \\
\hline $\mathbb{Z}_{2} \times \mathbb{Z}_{2}$ & $\mathrm{SU}(2)^{6}$ & 3 & 48 & 3 & 0 \\
\hline $\mathbb{Z}_{2} \times \mathbb{Z}_{4}$ & $\mathrm{SU}(2)^{2} \times \mathrm{SO}(5)^{2}$ & 3 & 58 & 1 & 0 \\
\hline $\mathbb{Z}_{2} \times \mathbb{Z}_{6}$ & $\mathrm{SU}(2)^{2} \times \mathrm{SU}(3) \times G_{2}$ & 3 & 48 & 1 & 2 \\
\hline $\mathbb{Z}_{2} \times \mathbb{Z}_{6^{\prime}}$ & $\mathrm{SU}(3) \times G_{2}^{2}$ & 3 & 33 & 0 & 0 \\
\hline $\mathbb{Z}_{3} \times \mathbb{Z}_{3}$ & $\mathrm{SU}(3)^{3}$ & 3 & 81 & 0 & 0 \\
\hline $\mathbb{Z}_{3} \times \mathbb{Z}_{6}$ & $\mathrm{SU}(3) \times G_{2}^{2}$ & 3 & 70 & 0 & 1 \\
\hline $\mathbb{Z}_{4} \times \mathbb{Z}_{4}$ & $\mathrm{SO}(5)^{3}$ & 3 & 87 & 0 & 0 \\
\hline $\mathbb{Z}_{6} \times \mathbb{Z}_{6}$ & $G_{2}^{3}$ & 3 & 81 & 0 & 0 \\
\hline
\end{tabular}

Table 1. Hodge number on toroidal orbifolds [29, 30]. 
Let us study the holomorphic $27^{3}$ Yukawa coupling associated with the untwisted Kähler moduli on $T^{6} / \mathbb{Z}_{7,8,12}$ and $T^{6} /\left(\mathbb{Z}_{N} \times \mathbb{Z}_{M}\right)$ orbifolds, on which the non-vanishing triple intersection number is evaluated as

$$
\kappa_{123}=1
$$

Hence, the untwisted Kähler moduli space on these toroidal orbifolds is described by the special Kähler manifold with $\left(\frac{\mathrm{SU}(1,1)}{\mathrm{U}(1)}\right)^{3}$. When we identify the Higgs field and three generations of quarks/leptons with, for instance, the elements of $A^{1}$ and $A^{1,2,3}$ associated with the untwisted Kähler moduli, the holomorphic Yukawa couplings of quarks and leptons become the rank-two matrix. There is no significant difference between the physical Yukawa couplings and the holomorphic one as analyzed before. In this way, it is difficult to realize the hierarchical structure of Yukawa couplings from the untwisted Kähler moduli. Similar phenomena occur in the $\overline{27}^{3}$ Yukawa couplings associated with the untwisted complex structure moduli on all the toroidal orbifolds whose Kähler potential is enumerated in eqs. (2.20)-(2.22).

One of the possibilities to realize the difference between the holomorphic Yukawa couplings and the physical one is to incorporate the off-diagonal Kähler metric of matter fields. The matter kinetic mixings are sourced by (i) the unfactorizable toroidal orbifolds such as $\mathbb{Z}_{3,4,6}$ orbifolds and/or (ii) the twisted modes in the effective action. In the next section, we study both possibilities to clarify the crucial role of off-diagonal entries in matter field Kähler metric. Before concluding this section, we exhibit a constraint on the moduli Kähler metric in the analysis of $27^{3}$ Yukawa couplings.

In the context of heterotic string theory, the value of $4 \mathrm{D}$ gauge coupling is directly related to the size of internal volume. We recall that the ten-dimensional (10D) heterotic supergravity action in string frame for our interest is of the form:

$$
S=\frac{M_{10}^{8}}{2} \int d^{10} x \sqrt{-G} e^{-2 \phi} R-\frac{1}{2 g_{10}^{2}} \int d^{10} x \sqrt{-G} e^{-2 \phi} \operatorname{Tr} F^{2}
$$

where $F$ denotes the gauge field strength of $E_{8} \times E_{8}$ or $\mathrm{SO}(32)$ gauge group with the adjoint representation. The $10 \mathrm{D}$ gravitational and gauge couplings are related as

$$
M_{10}^{8}=\frac{4 \pi}{l_{s}^{8}}=\frac{4}{\alpha^{\prime} g_{10}^{2}},
$$

with the string length $l_{s}=2 \pi \sqrt{\alpha^{\prime}}$. When we compactify the theory on a $6 \mathrm{D}$ internal manifold $\mathcal{M}$ with the volume $\operatorname{Vol}(\mathcal{M})=\mathcal{V} l_{s}^{6}$ measured in units of the string length, the $4 \mathrm{D}$ gauge coupling is determined by the internal volume $\mathcal{V}$ and the string coupling $g_{s}=e^{\langle\phi\rangle}$,

$$
g_{4}^{-2}=e^{-2\langle\phi\rangle} \operatorname{Vol}(\mathcal{M}) g_{10}^{-2}=\frac{\mathcal{V}}{4 \pi g_{s}^{2}} .
$$

It indicates that the internal volume should obey

$$
\mathcal{V}=g_{s}^{2} \alpha^{-1} \lesssim 25
$$


where we impose the small string coupling $g_{s} \leq 1$ and the $4 \mathrm{D}$ gauge coupling $\alpha^{-1}=$ $4 \pi g_{4}^{-2} \simeq 25$ required in supersymmetric SU(5) grand unified theory. Supersymmetry breaking at a high energy scale may allow larger $\alpha^{-1}$. In the following analysis, we allow for $\mathcal{V} \leq 30$ to be applicable to a broad class of $4 \mathrm{D}$ low-energy models. ${ }^{6}$ A strong correlation between the 4D gauge coupling and the size of internal volume is well known in a global model such as the heterotic string theory, but we will argue that the limited range of internal volume also constrain the structure of physical $27^{3}$ Yukawa couplings as discussed in detail later.

\section{Hierarchical structure of physical Yukawa couplings}

In this section, we explicitly demonstrate the hierarchical structure of physical Yukawa couplings on three backgrounds such as (i) $T^{6} / \mathbb{Z}_{3}$ orbifold without twisted modes in section 3.1, (ii) $T^{6} /\left(\mathbb{Z}_{2} \times \mathbb{Z}_{2}\right)$ orbifold with twisted modes in section 3.2, (iii) a mirror dual of $T^{6} /\left(\mathbb{Z}_{3} \times \mathbb{Z}_{3}\right)$ orbifold with twisted modes in section 3.3 , respectively.

\section{$3.1 \quad T^{6} / \mathbb{Z}_{3}$ orbifold}

For the first illustrative example, we deal with the $T^{6} / \mathbb{Z}_{3}$ orbifold, where the moduli fields on $T^{6} / \mathbb{Z}_{3}$ geometry consist of 9 untwisted and 27 twisted Kähler moduli. It is known that the Kähler potential of the untwisted Kähler moduli described by the $\frac{\mathrm{SU}(3,3)}{\mathrm{SU}(3) \times \mathrm{SU}(3) \times \mathrm{U}(1)}$ coset space is provided by

$$
K_{\mathrm{ks}}=-\ln \operatorname{det}(T+\bar{T})
$$

with

$$
T=\left(\begin{array}{lll}
T^{1} & T^{4} & T^{5} \\
T^{7} & T^{2} & T^{6} \\
T^{8} & T^{9} & T^{3}
\end{array}\right),
$$

and the corresponding triple intersection numbers are given by

$$
\kappa_{123}=\kappa_{468}=\kappa_{579}=1, \quad \kappa_{169}=\kappa_{258}=\kappa_{347}=-1,
$$

and 0 otherwise. The matter Käher potential and corresponding metric are also known as [37]

$$
\begin{aligned}
K & =-\ln \operatorname{det}\left(T+\bar{T}-A^{\alpha} A^{\bar{\alpha}}\right)^{a \bar{b}}=-\ln \operatorname{det}(T+\bar{T})^{a \bar{b}}+(T+\bar{T})_{a \bar{b}}^{-1} A_{\alpha}^{a} A_{\bar{\alpha}}^{\bar{b}}+\mathcal{O}\left(|A|^{4}\right), \\
K_{a \bar{b}}^{(27)} & =(T+\bar{T})_{a \bar{b}}^{-1},
\end{aligned}
$$

where $\alpha$ denotes the indices of $\mathrm{SU}(3) \subset E_{8}$. Note that the indices of $\alpha$ and $a$ are not identified with each other. The superpotential takes the form ${ }^{7}$

$$
W=\kappa_{a b c} \epsilon^{\alpha \beta \gamma} A_{\alpha}^{a} A_{\beta}^{b} A_{\gamma}^{c},
$$

\footnotetext{
${ }^{6}$ See ref. [36] for one-loop contributions to the gauge couplings in phenomenologically promising models from heterotic string theory.

${ }^{7}$ Since we are interested in the realization of hierarchical structure of physical Yukawa couplings, we simply omit the overall factor of the superpotential in the subsequent calculations.
} 
where $\kappa_{a b c}$ and $\epsilon^{\alpha \beta \gamma}$ correspond to the anti-symmetric tensors for $\mathrm{SU}(3)$ isometry and $\mathrm{SU}(3) \subset E_{8}$, respectively.

In contrary to the previous analysis, there exist the non-vanishing matter kinetic mixing. To examine the effects of off-diagonal entries in matter Kähler metric to the physical Yukawa couplings, we focus on $27^{3}$ Yukawa couplings of untwisted modes $\left\{A_{1}^{1}, A_{2}^{2}, A_{3}^{3}\right\}$ by assuming that they are protected from becoming massive. Note that the following analysis is applicable to other untwisted modes and we rewrite $\left\{A_{1}^{1}, A_{2}^{2}, A_{3}^{3}\right\}$ as $\left\{A^{1}, A^{2}, A^{3}\right\}$ to shorten the notation. Furthermore, we restrict ourselves on the locus $T^{4}=T^{5}=T^{6}=$ $T^{7}=T^{8}=T^{9}$ to simplify our analysis. On this locus, the moduli Kähler potential and matter Kähler metric are evaluated as follows:

$$
\begin{gathered}
K_{\mathrm{ks}}=-\ln \left[\left(T^{1}+\bar{T}^{1}\right)\left(T^{2}+\bar{T}^{2}\right)\left(T^{3}+\bar{T}^{3}\right)-\sum_{a=1}^{3}\left(T^{a}+\bar{T}^{a}\right)\left(T^{4}+\bar{T}^{4}\right)^{2}+2\left(T^{4}+\bar{T}^{4}\right)^{3}\right], \\
K_{a \bar{b}}^{(27)}=\frac{1}{2 t^{1} t^{2} t^{3}-2\left(t^{1}+t^{2}+t^{3}\right)\left(t^{4}\right)^{2}+4\left(t^{4}\right)^{3}}\left(\begin{array}{ccc}
t^{2} t^{3}-\left(t^{4}\right)^{2} & \left(t^{4}-t^{3}\right) t^{4} & \left(t^{4}-t^{2}\right) t^{4} \\
\left(t^{4}-t^{3}\right) t^{4} & t^{1} t^{3}-\left(t^{4}\right)^{2} & \left(t^{4}-t^{1}\right) t^{4} \\
\left(t^{4}-t^{2}\right) t^{4} & \left(t^{4}-t^{1}\right) t^{4} & t^{1} t^{2}-\left(t^{4}\right)^{2}
\end{array}\right),
\end{gathered}
$$

with $t^{a}=\operatorname{Re}\left(T^{a}\right)$, which reduces to the diagonal matter Kähler metric in the $t^{4} \rightarrow 0$ limit, namely the value of $t^{4}$ controls whether the Kähler metric is diagonal.

When the vacuum expectation values of the untwisted Kähler moduli are restricted along the isotropic locus,

$$
\left\langle t^{1}\right\rangle=\left\langle t^{2}\right\rangle=\left\langle t^{3}\right\rangle=x, \quad\left\langle t^{4}\right\rangle=x^{p}
$$

with $x>1$ and $p \geq 0,{ }^{8}$ eigenvalues of the moduli Kähler metric are simply obtained as

$$
\Lambda_{\hat{a} \hat{b}}=\left(\begin{array}{ccc}
\left(2 x-2 x^{p}\right)^{-1} & 0 & 0 \\
0 & \left(2 x-2 x^{p}\right)^{-1} & 0 \\
0 & 0 & \left(2 x+4 x^{p}\right)^{-1}
\end{array}\right)
$$

by employing the following diagonalizing matrix of moduli Kähler metric:

$$
L=\left(\begin{array}{ccc}
-1 & 0 & 1 \\
-1 & 1 & 0 \\
1 & 1 & 1
\end{array}\right)
$$

The physical Yukawa couplings are then computed by substituting these into the formula (2.12). It turns out that the physical Yukawa couplings $Y_{\hat{1} \hat{a} \hat{b}}$ and $Y_{\hat{2} \hat{a} \hat{b}}$ become the rank-two matrices due to the presence of degenerate eigenvalues in the Kähler metric (3.8). For that reason, we assume that the Higgs field is originating from $A^{\hat{3}}$, and quarks/leptons

\footnotetext{
${ }^{8}$ We restrict ourselves to these moduli space, otherwise the value of $t^{1,2,3,4}$ can be smaller than 1 in contradiction with the reliability of the supergravity description.
} 
are the elements of $\left(A^{\hat{1}}, A^{\hat{2}}, A^{\hat{3}}\right)$, respectively. Indeed, from an analytical expression of physical Yukawa couplings $Y_{\hat{a} \hat{a} \hat{b}}$,

$$
\begin{aligned}
Y_{\hat{\mathrm{a}} \hat{a} \hat{b}} & =e^{K_{\mathrm{ks}}} \sum_{d, e, f=1}^{3}\left(\Lambda^{-1 / 2} L\right)_{\hat{3}}^{d}\left(\Lambda^{-1 / 2} L\right)_{\hat{b}}^{e}\left(\Lambda^{-1 / 2} L\right)_{\hat{c}}^{f} \kappa_{\text {def }} \\
& =e^{K_{\mathrm{ks}}}(2 \sqrt{2})^{-1}\left(x+2 x^{p}\right)^{-1 / 2}\left(\begin{array}{ccc}
2\left(-x+x^{p}\right) & -x+x^{p} & 0 \\
-x+x^{p} & 2\left(-x+x^{p}\right) & 0 \\
0 & 0 & 6\left(x+2 x^{p}\right)
\end{array}\right),
\end{aligned}
$$

the determinant

$$
\operatorname{det}\left(Y_{\hat{a} \hat{a} \hat{b}}\right)=e^{3 K_{\mathrm{ks}}} 288 \sqrt{2}\left(x-x^{p}\right)^{2}\left(x+2 x^{p}\right)^{5 / 2}
$$

turns out to be non-zero unless $p=0$. One can realize the rank-three physical Yukawa matrix unless $p=0$, although the holomorphic Yukawa coupling (2.16) is rank-two matrix. Hence, off-diagonal entries in the matter field Kähler metric bring us a rich structure of the physical Yukawa couplings. In the current parametrization of the moduli fields (3.7), eigenvalues of $Y_{\hat{a} \hat{a} \hat{b}}$ are calculated as

$$
\begin{aligned}
& m_{1}=e^{K_{\mathrm{ks}}} 12 \sqrt{2}\left(x+2 x^{p}\right)^{3 / 2}, \\
& m_{2}=e^{K_{\mathrm{ks}}} 6 \sqrt{2}\left(-x+x^{p}\right)\left(x+2 x^{p}\right)^{1 / 2}, \\
& m_{3}=e^{K_{\mathrm{ks}}} 2 \sqrt{2}\left(-x+x^{p}\right)\left(x+2 x^{p}\right)^{1 / 2},
\end{aligned}
$$

with the order $\left|m_{1}\right|>\left|m_{2}\right|>\left|m_{3}\right|$ and these ratios become

$$
r_{1}=\frac{\left|m_{2}\right|}{\left|m_{1}\right|}=\frac{\left|x-x^{p}\right|}{2\left(x+2 x^{p}\right)}, \quad r_{2}=\frac{\left|m_{3}\right|}{\left|m_{1}\right|}=\frac{\left|x-x^{p}\right|}{6\left(x+2 x^{p}\right)},
$$

satisfying $\left|m_{3}\right| /\left|m_{2}\right|=1 / 3$. Taking into account the constraint for the internal volume

$$
\mathcal{V}=8\left(x-x^{p}\right)^{2}\left(x+2 x^{p}\right) \leq 30,
$$

it turns out that the typical ratio $\left|m_{3}\right| /\left|m_{1}\right|$ is of $\mathcal{O}(0.01)$ to be consistent with the realistic value of $4 \mathrm{D}$ gauge coupling.

To exemplify the realization of the hierarchical physical Yukawa couplings, we search the anisotropic untwisted Kähler moduli by changing the moduli locus (3.7) to

$$
\left\langle t^{1}\right\rangle=\left\langle t^{2}\right\rangle=x, \quad\left\langle t^{3}\right\rangle=y, \quad\left\langle t^{4}\right\rangle=z,
$$

with $x, y, z>1$. When we adopt the above parametrization of the moduli fields, the moduli Kähler metric (3.6) is diagonalized as follows:

$$
\Lambda_{\hat{a} \hat{b}}=\left(\begin{array}{ccc}
\frac{1}{2(x-z)} & 0 & 0 \\
0 & \Lambda_{-}^{-1} & 0 \\
0 & 0 & \Lambda_{+}^{-1}
\end{array}\right),
$$



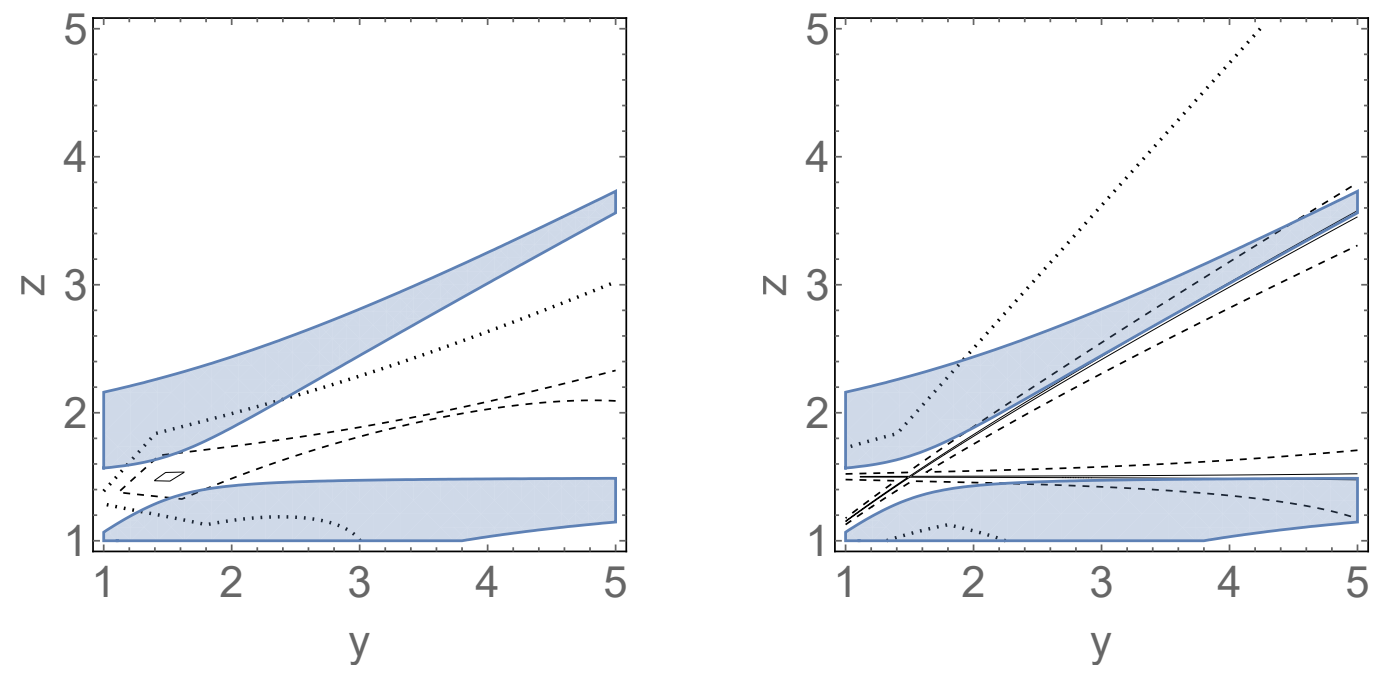

Figure 1. In the left and right panels, we plot two ratios $r_{1}$ and $r_{2}$ of eigenvalues of the physical Yukawa couplings $\left(Y_{\hat{a} \hat{a} \hat{b}}\right)$ to the maximum one at the fixed $x=1.5$, respectively. The dotted, dashed, and solid curves correspond to the values for $10^{-1}\left(10^{-1}\right), 0.05\left(10^{-2}\right)$, and $10^{-2}\left(10^{-3}\right)$, in the left (right) panel. In both panels, blue shaded regions represent the allowed volume $1<\mathcal{V} \leq 30$. Note that the Yukawa matrix reduces to the rank-two Yukawa matrix at $z=\frac{y+\sqrt{y(8 x+y)}}{4}$ and $z=x$, in the vicinity of the solid curves.

with

$$
\Lambda_{ \pm}=x+y+z \pm \sqrt{x^{2}+y^{2}+2 x z+9 z^{2}-2 y(x+z)} .
$$

Here, the diagonalization of the Kähler metric is performed by

$$
L=\left(\begin{array}{lll}
-1 & 1 & 0 \\
L_{+} & L_{+} & 1 \\
L_{-} & L_{-} & 1
\end{array}\right)
$$

with

$$
L_{ \pm}=\frac{x-y+z \pm \sqrt{x^{2}+y^{2}+2 x z+9 z^{2}-2 y(x+z)}}{4 z} .
$$

We arrive at the physical Yukawa couplings by substituting these quantities into eq. (2.12). Since the analytical expression of the physical Yukawa couplings is complicated with respect to the moduli fields, we rely on the numerical search to find out desirable hierarchical structure of physical Yukawa couplings. In particular, we focus on physical Yukawa coupling $Y_{\hat{3} \hat{a} \hat{b}}$, setting one of moduli fields at specific values. Note that $Y_{\hat{1} \hat{a} \hat{b}}$ becomes the rank-two matrix and $Y_{\hat{2} \hat{a} \hat{b}}$ has the same structure as $Y_{\hat{a} \hat{a} \hat{b}}$ due to the structure of diagonalizing matrix (3.17).

We plot two ratios of eigenvalues of the physical Yukawa couplings $\left(Y_{\hat{3} \hat{a} \hat{b}}\right)$ to the maximum one in the left panel for $r_{1}$ and the right panel for $r_{2}$ in figure 1 with $x=1.5$ and figure 2 with $x=2$. In contrast to the case with isotropic untwisted Kähler moduli, the solid curves in figures 1 and 2 show that $\mathcal{O}(1)$ values of the moduli fields induce the $\mathcal{O}\left(10^{-2}\right)$ and $\mathcal{O}\left(10^{-3}\right)$ hierarchical structures of the physical Yukawa couplings for $r_{1}$ 

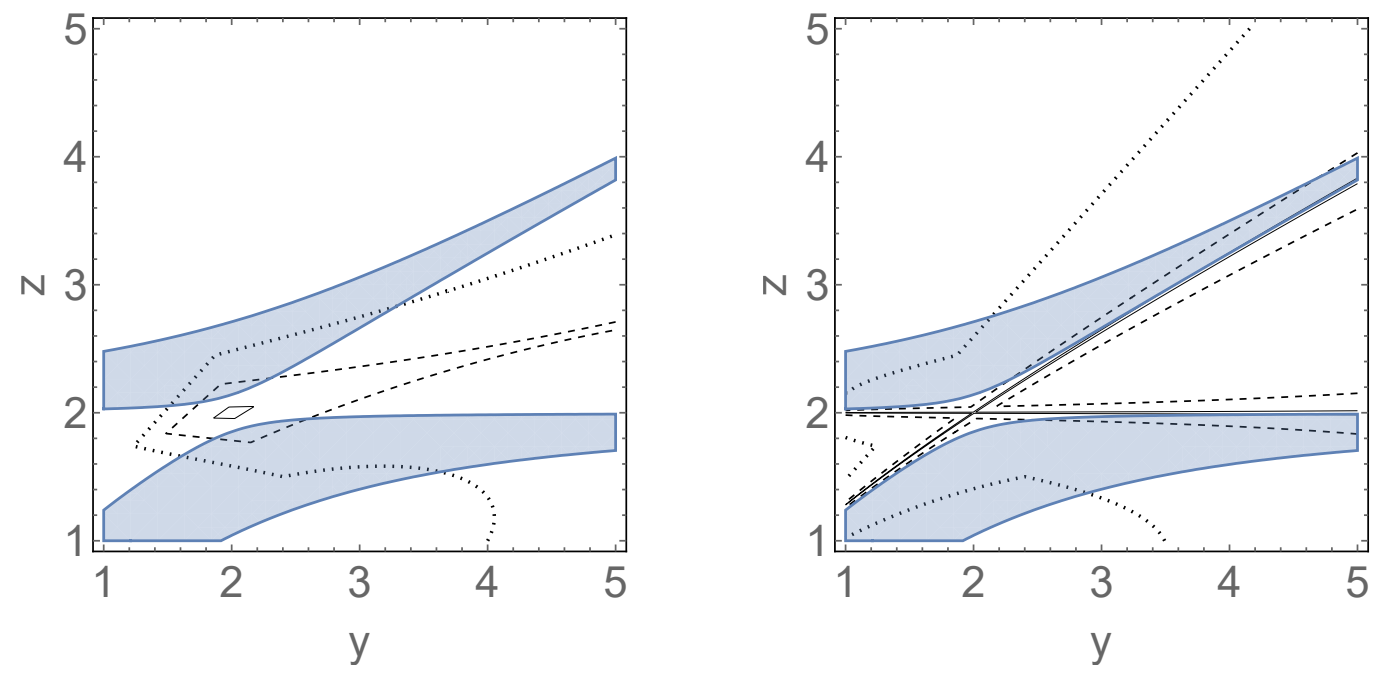

Figure 2. In the left and right panels, we plot two ratios $r_{1}$ and $r_{2}$ of eigenvalues of the physical Yukawa couplings $\left(Y_{\hat{3} \hat{a} \hat{b}}\right)$ to the maximum one at the fixed $x=2$, respectively. The dotted, dashed, and solid curves correspond to the values for $10^{-1}\left(10^{-1}\right), 0.05\left(10^{-2}\right)$, and $10^{-2}\left(10^{-3}\right)$, in the left (right) panel. In both panels, blue shaded regions represent the allowed volume $1<\mathcal{V} \leq 30$. Note that the Yukawa matrix reduces to the rank-two Yukawa matrix at $z=\frac{y+\sqrt{y(8 x+y)}}{4}$ and $z=x$, in the vicinity of the solid curves.

\begin{tabular}{|c|c|c|}
\hline$(x, y, z)$ & $\left(1, r_{1}, r_{2}\right)$ & $\mathcal{V}$ \\
\hline$(1.5,4,3.2)$ & $\left(1,0.15,1.1 \times 10^{-2}\right)$ & 22.8 \\
\hline$(1.5,5,3.7)$ & $\left(1,0.15,6.0 \times 10^{-3}\right)$ & 24.3 \\
\hline$(2,4.5,3.7)$ & $\left(1,0.14,9.2 \times 10^{-3}\right)$ & 23.5 \\
\hline
\end{tabular}

Table 2. Benchmark ratios for the ratio $\left(1, r_{1}, r_{2}\right)$ and the toroidal volume $\mathcal{V}$ at several moduli values.

and $r_{2}$. A tuning of the moduli fields leads to the more hierarchical structure of physical Yukawa couplings, irrespective of the value $x$.

Remarkably, there exists an overlap region between the region with the hierarchical Yukawa couplings and the desired value of the toroidal volume $\mathcal{V} \leq 30$. Note that the volume of the toroidal orbifold $\mathcal{V}=8(x-z)(x y+(y-2 z) z)$ goes to zero when $y$ and $z$ approach to $x$. In table 2 , we show three benchmark values for the ratio $\left(1, r_{1}, r_{2}\right)$ at several values of the Kähler moduli.

\section{$3.2 \quad$ Blown-up $T^{6} /\left(\mathbb{Z}_{2} \times \mathbb{Z}_{2}\right)$ orbifold}

In this section, we discuss the $T^{6} /\left(\mathbb{Z}_{2} \times \mathbb{Z}_{2}\right)$ orbifold including twisted modes. On the $T^{6} /\left(\mathbb{Z}_{2} \times \mathbb{Z}_{2}\right)$ geometry, the Kähler metric of matter fields associated with the untwisted Kähler moduli is factorizable and their physical Yukawa couplings have the same structure of the holomorphic Yukawa couplings. Hence, we study in detail the impacts of twisted Kähler moduli on the physical Yukawa coupling. 
The Kähler form on the blown-up $T^{6} /\left(\mathbb{Z}_{2} \times \mathbb{Z}_{2}\right)$ geometry is expanded as

$$
J=\sum_{a=1}^{3} t^{a} R_{a}-\sum_{r=1}^{48} s^{r} E_{r}
$$

where $t^{a}$ denotes the untwisted Kähler moduli associated with divisors $R_{a}$ represented as $d z_{i} \wedge d \bar{z}_{i}$ using the complex coordinates of tori $z_{i}$, whereas $s^{r}$ denote the twisted Kähler moduli associated with exceptional divisors $E_{r}$. Note that $t^{a}$ and $s^{r}$ are chosen to be positive to give a geometrical interpretation, and furthermore $t^{a}$ should be larger than $s^{r}$ to ensure the positivity of curves, divisors and entire volume.

In this paper, we restrict ourselves to the simplified case, where all the twisted Kähler moduli are identified with

$$
t^{4} \equiv s^{r}, \quad(r=1,2, \cdots, 48) .
$$

Here and in what follows, we follow the result in eq. (6.8) of ref. [38], although there exists a huge possibility of resolutions. (For more details, see, refs. [38, 39].) The Kähler potential on resolutions of the blown-up $T^{6} /\left(\mathbb{Z}_{2} \times \mathbb{Z}_{2}\right)$ orbifold is given by

$$
K=-\ln \left[\frac{1}{8}\left(T^{1}+\bar{T}^{1}\right)\left(T^{2}+\bar{T}^{2}\right)\left(T^{3}+\bar{T}^{3}\right)-\sum_{a=1}^{3}\left(T^{a}+\bar{T}^{a}\right)\left(T^{4}+\bar{T}^{4}\right)^{2}+6\left(T^{4}+\bar{T}^{4}\right)^{3}\right],
$$

with $t^{a}=\operatorname{Re}\left(T^{a}\right)$, approaching to the volume of $T^{6} /\left(\mathbb{Z}_{2} \times \mathbb{Z}_{2}\right)$ orbifold in the limit $t^{1,2,3} \gg$ $t^{4}$. Here the effective triple intersection numbers are

$$
\kappa_{123}=1, \quad \kappa_{a 44}=-16, \quad \kappa_{444}=288,
$$

with $a=1,2,3$, and 0 otherwise. The inclusion of the blow-up modes gives rise to the matter kinetic mixing. Indeed, the Kähler potential has a similar structure to $T^{6} / \mathbb{Z}_{3}$ orbifold with the identification $T^{4}=T^{5}=T^{6}=T^{7}=T^{8}=T^{9}$ as shown in eq. (3.6), where only the difference is the coefficient of $\left(T^{4}+\bar{T}^{4}\right)$. In the following subsections, we assume the specific modes protected becoming massive to reveal the structure of physical Yukawa couplings. In particular, we analyze two types of Yukawa couplings; (i) three-point couplings of untwisted modes themselves in section 3.2.1 and (ii) three-point couplings of (untwisted)-(untwisted)-(twisted) modes in section 3.2.2.

\subsubsection{Yukawa couplings of untwisted modes}

To analyze the structure of physical Yukawa couplings of untwisted modes themselves, we search the anisotropic untwisted Kähler moduli:

$$
\left\langle t^{1}\right\rangle=\left\langle t^{2}\right\rangle=x, \quad\left\langle t^{3}\right\rangle=y, \quad\left\langle t^{4}\right\rangle=z,
$$

with $x, y, z>1$. When we adopt the above parametrization of the moduli fields, the moduli Kähler metric is calculated as

$$
\Lambda_{\hat{a} \hat{b}}^{\left(\mathbb{Z}_{2} \times \mathbb{Z}_{2}\right)}=\frac{1}{8\left(x^{2} y-8(2 x+y) z^{2}+48 z^{3}\right)^{2}}\left(\begin{array}{ccc}
2 y\left(x^{2} y-8(2 x+y) z^{2}+48 z^{3}\right) & 0 & 0 \\
0 & \Lambda_{-}^{\left(\mathbb{Z}_{2} \times \mathbb{Z}_{2}\right)} & 0 \\
0 & 0 & \Lambda_{+}^{\left(\mathbb{Z}_{2} \times \mathbb{Z}_{2}\right)}
\end{array}\right),
$$


with

$$
\begin{aligned}
\Lambda_{ \pm}^{\left(\mathbb{Z}_{2} \times \mathbb{Z}_{2}\right)}= & x^{4}-16 x y z^{2}+x^{2}\left(y^{2}-16 z^{2}\right)+8 z^{2}\left(y^{2}-6 y z+24 z^{2}\right) \pm \sqrt{\Lambda^{\left(\mathbb{Z}_{2} \times \mathbb{Z}_{2}\right)}}, \\
\Lambda^{\left(\mathbb{Z}_{2} \times \mathbb{Z}_{2}\right)} \equiv & -2048 x(3 x-8 z)(x-3 z) z^{5}+64 y z^{2}(x+3 z)\left(x^{2}-8 z^{2}\right)^{2}-4 y^{2}\left(x^{2}-8 z^{2}\right)^{2}\left(x^{2}+8 z^{2}\right) \\
& +\left(x^{4}-16 x^{2} z^{2}+192 z^{4}-16 y z^{2}(x+3 z)+y^{2}\left(x^{2}+8 z^{2}\right)\right)^{2} .
\end{aligned}
$$

Here, the diagonalization of the Kähler metric is performed by

$$
L^{\left(\mathbb{Z}_{2} \times \mathbb{Z}_{2}\right)}=\left(\begin{array}{ccr}
-1 & 1 & 0 \\
L_{1}-\frac{\Lambda_{+}^{\left(\mathbb{Z}_{2} \times \mathbb{Z}_{2}\right)}}{32 z^{2}\left(8 y^{2}-6 z x+x^{2}\right)} & L_{1}-\frac{\Lambda_{+}^{\left(\mathbb{Z}_{2} \times \mathbb{Z}_{2}\right)}}{32 z^{2}\left(8 y^{2}-6 z x+x^{2}\right)} & 1 \\
L_{1}-\frac{\Lambda_{-}^{\left(\mathbb{Z}_{2} \times \mathbb{Z}_{2}\right)}}{32 z^{2}\left(8 y^{2}-6 z x+x^{2}\right)} & L_{1}-\frac{\Lambda_{-}^{\left(\mathbb{Z}_{2} \times \mathbb{Z}_{2}\right)}}{32 z^{2}\left(8 y^{2}-6 z x+x^{2}\right)} & 1
\end{array}\right),
$$

with

$$
L_{1} \equiv \frac{x^{2} y^{2}+8 y(-2 x+y) z^{2}-48 y z^{3}+128 z^{4}}{16(x-4 z)(x-2 z) z^{2}} .
$$

We arrive at the physical Yukawa couplings by substituting these quantities into eq. (2.12). In a similar way with analysis on $T^{6} / \mathbb{Z}_{3}$ orbifold, we perform the numerical search to clarify the structure of physical Yukawa couplings by setting $x=4$. Since the value of blow-up mode has to be smaller than the other moduli values to justify our effective action, we choose a relatively large value for $x$, that is, $x=4$. We find that physical Yukawa coupling $Y_{\hat{1} \hat{a} \hat{b}}$ becomes the rank-two matrix due to the structure of diagonalizing matrix (3.25). In this respect, the Higgs field is assumed to either elements of $A^{2}$ or $A^{3}$, and quarks/leptons are the elements of $\left(A^{1}, A^{2}, A^{3}\right)$.

In figures 3 and 4 , we plot two ratios of eigenvalues of the physical Yukawa couplings $Y_{\hat{2} \hat{a} \hat{b}}$ and $Y_{\hat{3} \hat{a} \hat{b}}$ to the maximum one,

$$
r_{1}=\frac{\left|m_{2}\right|}{\left|m_{1}\right|}, \quad r_{2}=\frac{\left|m_{3}\right|}{\left|m_{1}\right|}
$$

with the order $\left|m_{1}\right|>\left|m_{2}\right|>\left|m_{3}\right|$, respectively. The left and right panels in figures 3 and 4 correspond to the $r_{1}$ and $r_{2}$ with respect to $y$ and $z$, respectively. In the same way as the results on $T^{6} / \mathbb{Z}_{3}$ orbifold, $\mathcal{O}(1)$ values of the moduli fields induce the $\mathcal{O}\left(10^{-1}\right)$ and $\mathcal{O}\left(10^{-2}\right)$ hierarchical structures for $r_{1}$ and $r_{2}$, because of the existence of blow-up modes appearing in the off-diagonal entries of matter Kähler metric. Remarkably, there also exists an overlap region between the region with the hierarchical Yukawa couplings and the desired value of the toroidal volume $\mathcal{V} \leq 30$.

\subsubsection{Yukawa couplings of the twisted mode}

In this section, we analyze the Yukawa couplings among two untwisted modes and the twisted mode by assuming that the matter fields in the SM are originating from the untwisted modes $\left(A^{1}, A^{2}, A^{3}\right)$ and the twisted mode $A^{4}$. Since the holomorphic Yukawa coupling as well as the Kähler metric of the untwisted modes $\left(A^{1}, A^{2}, A^{3}\right)$ is totally symmetric, we focus on the Yukawa coupling of $\left(A^{1}, A^{2}, A^{4}\right)$ without loss of generality. The 

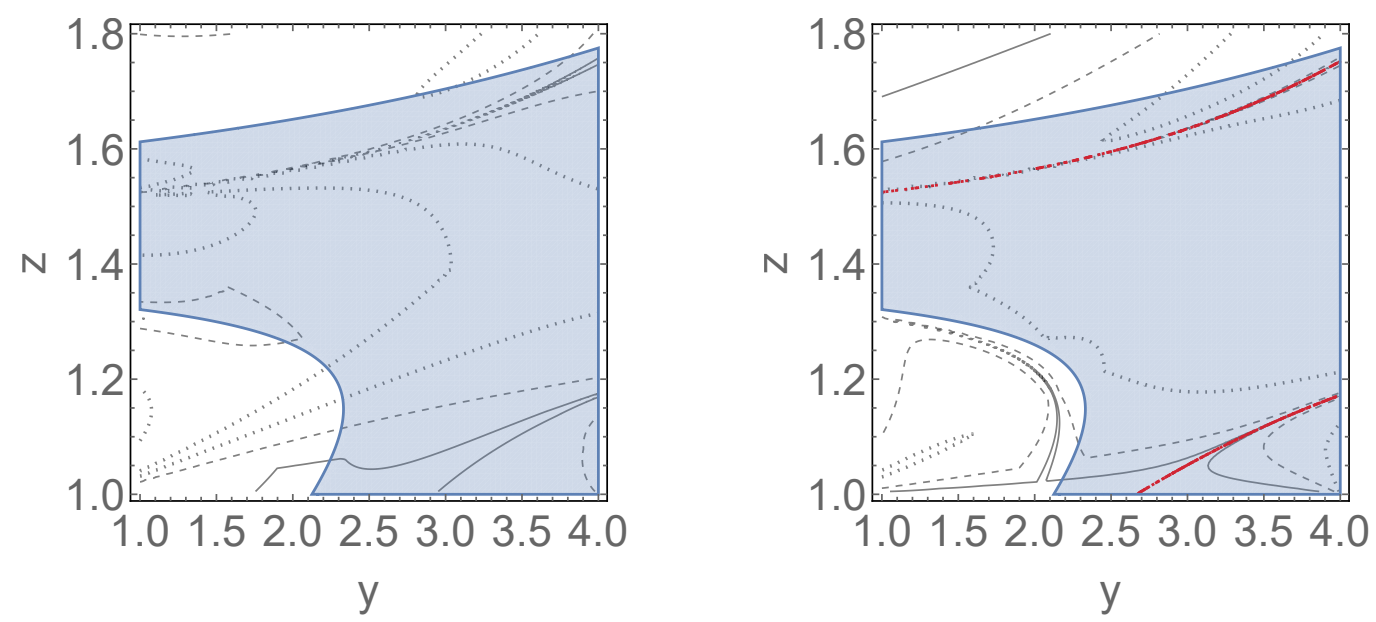

Figure 3. In the left and right panels, we plot two ratios $r_{1}$ and $r_{2}$ of eigenvalues of the physical Yukawa couplings $\left(Y_{\hat{2} \hat{a} \hat{b}}\right)$ to the maximum one with respect to $y$ and $z$, respectively. The dotted, dashed, and solid curves correspond to the values for $0.5\left(10^{-1}\right), 10^{-1}\left(10^{-2}\right)$, and $10^{-2}\left(10^{-3}\right)$, in the left (right) panel. Note that Yukawa couplings reduce to the rank-two Yukawa matrix on red curves in the right panel. In both panels, the blue shaded regions represent the allowed volume $1<\mathcal{V} \leq 30$.
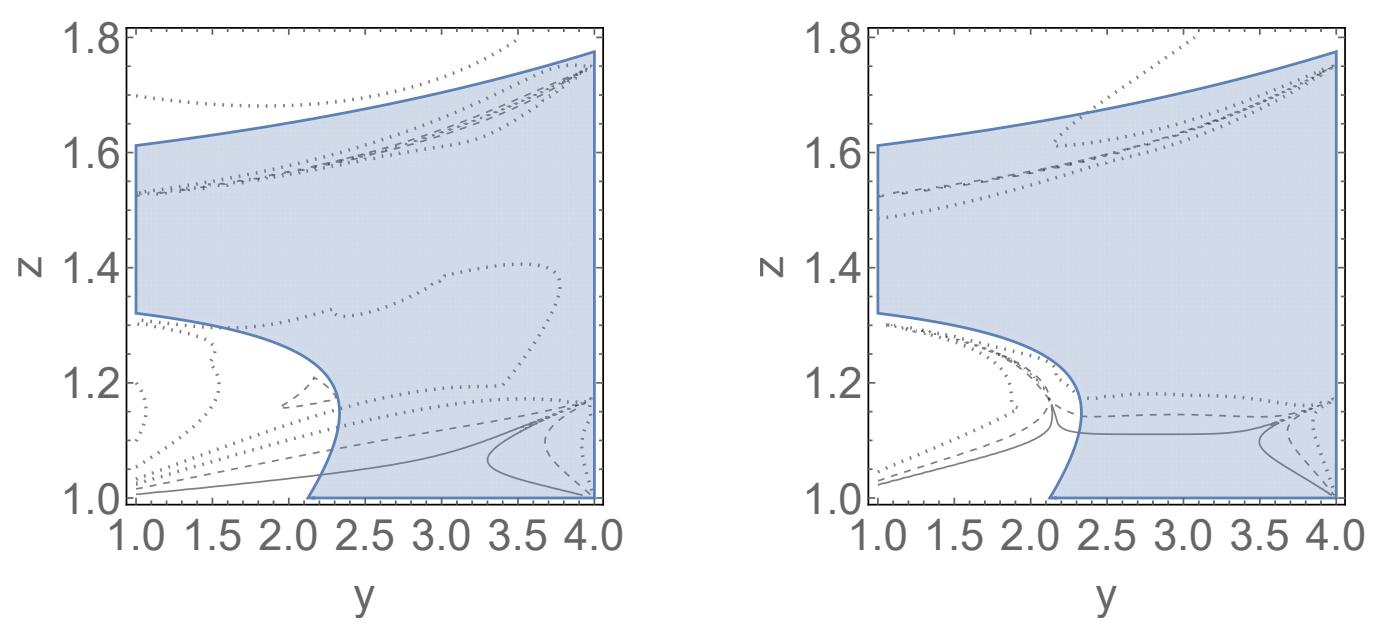

Figure 4. In the left and right panels, we plot two ratios $r_{1}$ and $r_{2}$ of eigenvalues of the physical Yukawa couplings $\left(Y_{\hat{z} \hat{a} \hat{b}}\right)$ to the maximum one with respect to $y$ and $z$, respectively. The dotted, dashed, and solid curves correspond to the values for $0.5\left(10^{-1}\right), 10^{-1}\left(10^{-2}\right)$, and $10^{-2}\left(10^{-3}\right)$, in the left (right) panel, respectively. In both panels, the blue shaded regions represent the allowed volume $1<\mathcal{V} \leq 30$.

holomorphic Yukawa couplings are of the form

$$
\kappa_{1 a b}=\kappa_{2 a b}=\left(\begin{array}{ccc}
0 & 0 & 0 \\
0 & 0 & 0 \\
0 & 0 & -16
\end{array}\right), \quad \kappa_{4 a b}=\left(\begin{array}{ccc}
0 & 0 & -16 \\
0 & 0 & -16 \\
-16 & -16 & 288
\end{array}\right)
$$

which are rank 1 and 2 matrices, respectively. In the following, we examine the structure of physical Yukawa couplings with an emphasis on three classes of holomorphic Yukawa 


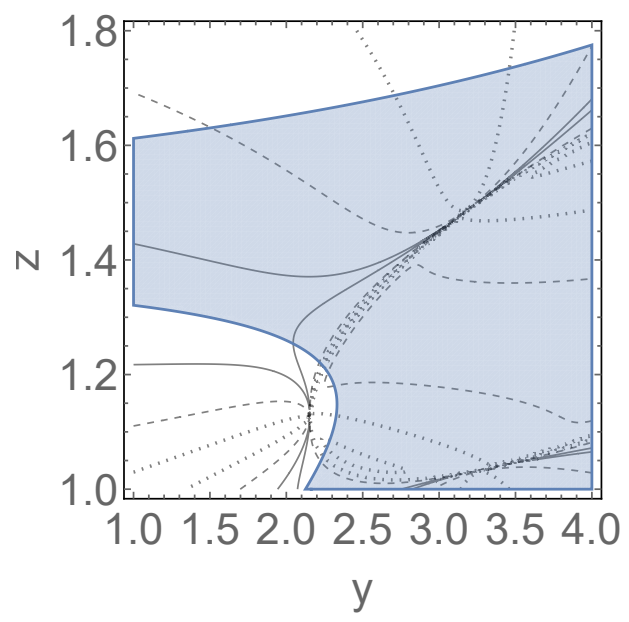

Figure 5. We draw the ratio of eigenvalues of the rank-two physical Yukawa couplings $\left(Y_{\hat{3} \hat{a} \hat{b}}\right)$ to the maximum one with respect to $y$ and $z$. The dotted, dashed, and solid curves correspond to the values for $10^{-1}, 10^{-2}$, and $10^{-3}$, and the blue shaded regions represent the allowed volume $1<\mathcal{V} \leq 30$.

couplings, namely (i) $\kappa_{1 a b}$, (ii) $\kappa_{2 a b}$, (iii) $\kappa_{4 a b}$, where the Higgs field is identified with the element of $A^{1}, A^{2}$ and $A^{4}$, respectively. In all cases, quarks/leptons are assumed to the elements of $\left(A^{1}, A^{2}, A^{4}\right)$.

The moduli Kähler metric is calculated as

$$
\begin{aligned}
& K_{1 \overline{1}}=\frac{\left(t^{2} t^{3}-8\left(t^{4}\right)^{2}\right)^{2}}{4\left(t^{1} t^{2} t^{3}-8\left(t^{1}+t^{2}+t^{3}\right)\left(t^{4}\right)^{2}+48\left(t^{4}\right)^{3}\right)^{2}}, \quad K_{2 \overline{2}}=\frac{\left(t^{1} t^{3}-8\left(t^{4}\right)^{2}\right)^{2}}{4\left(t^{1} t^{2} t^{3}-8\left(t^{1}+t^{2}+t^{3}\right)\left(t^{4}\right)^{2}+48\left(t^{4}\right)^{3}\right)^{2}} \\
& K_{4 \overline{4}}=\frac{16\left(t^{1} t^{2} t^{3}\left(t^{1}+t^{2}+t^{3}\right)-18 t^{1} t^{2} t^{3} t^{4}+8\left(t^{1}+t^{2}+t^{3}\right)^{2}\left(t^{4}\right)^{2}-96\left(t^{1}+t^{2}+t^{3}\right)\left(t^{4}\right)^{3}+432\left(t^{4}\right)^{4}\right)}{4\left(t^{1} t^{2} t^{3}-8\left(t^{1}+t^{2}+t^{3}\right)\left(t^{4}\right)^{2}+48\left(t^{4}\right)^{3}\right)^{2}} \\
& K_{1 \overline{2}}=K_{2 \overline{1}}=\frac{8\left(t^{3}-4 t^{4}\right)\left(t^{3}-2 t^{4}\right)\left(t^{4}\right)^{2}}{4\left(t^{1} t^{2} t^{3}-8\left(t^{1}+t^{2}+t^{3}\right)\left(t^{4}\right)^{2}+48\left(t^{4}\right)^{3}\right)^{2}}, \quad K_{1 \overline{4}}=K_{4 \overline{1}}=\frac{16 t^{4}\left(-t^{2} t^{3}\left(t^{2}+t^{3}\right)+9 t^{2} t^{3} t^{4}-24\left(t^{4}\right)^{3}\right)}{4\left(t^{1} t^{2} t^{3}-8\left(t^{1}+t^{2}+t^{3}\right)\left(t^{4}\right)^{2}+48\left(t^{4}\right)^{3}\right)^{2}} \\
& K_{2 \overline{4}}=K_{4 \overline{2}}=\frac{16 t^{4}\left(-t^{1} t^{3}\left(t^{1}+t^{3}\right)+9 t^{1} t^{3} t^{4}-24\left(t^{4}\right)^{3}\right)}{4\left(t^{1} t^{2} t^{3}-8\left(t^{1}+t^{2}+t^{3}\right)\left(t^{4}\right)^{2}+48\left(t^{4}\right)^{3}\right)^{2}}
\end{aligned}
$$

with $t^{a}=\operatorname{Re}\left(T^{a}\right)$ and hereafter, we adopt the parametrization of the moduli vacuum expectation values (3.23) in the same way as the previous analysis. We omit the complicated expressions for the eigenvalues of the Kähler metric as well as its diagonalization matrix. The physical Yukawa couplings are calculated from the formula

$$
Y_{\hat{a} \hat{b} \hat{c}}=e^{K_{\mathrm{ks}}} \sum_{d, e, f=1,2,4}\left(\Lambda^{-1 / 2} L\right)_{\hat{a}}^{d}\left(\Lambda^{-1 / 2} L\right)_{\hat{b}}^{e}\left(\Lambda^{-1 / 2} L\right)_{\hat{c}}^{f} \kappa_{d e f} .
$$

From our numerical search along the moduli locus (3.23), we find that the rank of physical Yukawa couplings in all three cases (i)-(iii) has two and there is no significant differences among them. Hence, we show the non-vanishing ratio of physical Yukawa coupling in the case (iii) as shown in figure 5 with $x=4$. The difficulty of realizing the full rank of physical Yukawa couplings would be attributable to the trivial structure of holomorphic Yukawa couplings. 


\subsection{A mirror dual of $T^{6} /\left(\mathbb{Z}_{3} \times \mathbb{Z}_{3}\right)$ orbifold}

As a final example, we deal with a mirror dual of rigid $T^{6} /\left(\mathbb{Z}_{3} \times \mathbb{Z}_{3}\right)$ orbifold as developed in ref. [40]. In contrary to the previous backgrounds, there exist $h^{2,1}=30$ number of complex structure deformation and no Kähler deformation. Hence, there will be no constraint on the internal volume that determines the value of $4 \mathrm{D}$ gauge coupling. The chiral zero-modes for our interest are originating from the complex structure moduli whose physical Yukawa couplings are analyzed in detail below.

In this section, we show the hierarchical structure about the physical Yukawa couplings for chiral zero-modes associated with the complex structure moduli. In particular, we focus on one of the blow-up modes $(\chi)$ among totally 27 blow-up modes, which is expanded in the small complex structure regime. On the other hand, the bulk complex structure moduli $U^{i}$ are considered the large complex structure regime, that is,

$$
\operatorname{Im}\left(U^{i}\right)>1, \quad|\chi|<1,
$$

with $i=1,2,3$. Note that we still call "blow-up modes" on a mirror dual of $T^{6} /\left(\mathbb{Z}_{3} \times \mathbb{Z}_{3}\right)$ orbifold, since they correspond to Kähler moduli associated with exceptional divisor on a rigid $T^{6} /\left(\mathbb{Z}_{3} \times \mathbb{Z}_{3}\right)$ orbifold. The moduli Kähler potential on a mirror dual of rigid $T^{6} /\left(\mathbb{Z}_{3} \times \mathbb{Z}_{3}\right)$ is known as

$$
K=-\ln \left[i\left(U^{1}-\bar{U}^{1}\right)\left(U^{2}-\bar{U}^{2}\right)\left(U^{3}-\bar{U}^{3}\right)\right]+\frac{27 \sqrt{3} \Gamma\left(\frac{1}{3}\right)^{6}}{(2 \pi)^{6}} \frac{|\chi|^{2}}{i\left(U^{1}-\bar{U}^{1}\right)\left(U^{2}-\bar{U}^{2}\right)\left(U^{3}-\bar{U}^{3}\right)},
$$

taking into account the regime (3.31). In addition, the holomorphic Yukawa couplings are also found by calculating the integrals of the holomorphic three-form over three-cycles. The non-vanishing holomorphic Yukawa couplings under the regime (3.31) are given by

$$
\kappa_{123}=1,
$$

where we omit an expression of exponentially-suppressed holomorphic Yukawa couplings of the blow-up mode. Since we are interested in assessing to what extent the Kähler mixing induces the hierarchical structure of physical Yukawa couplings, we focus on the Yukawa couplings of untwisted modes. It is remarkable that the blow-up mode is now expanded in the small complex structure regime and in the blow-down limit $\chi \rightarrow 0$, other holomorphic Yukawa couplings such as $\kappa_{\chi \chi U^{i}}$ and $\kappa_{\chi U^{i} U^{j}}$ vanish. We refer ref. [40] for the derivation of the moduli effective action, which can be achieved by calculating the holomorphic threeform of CY threefold embedded into the complex projective space $\mathbb{C P}^{8}$.

We are ready to analyze the physical Yukawa couplings (2.12) with the Kähler metric given by (3.32) and the holomorphic Yukawa couplings (3.33), in particular for the untwisted modes. The explicit form of the Kähler metric is given by

$$
K_{i \bar{j}}=\left(\begin{array}{ccc}
\frac{1}{4\left(\tau^{1}\right)^{2}}+\frac{27 \sqrt{3}|\chi|^{2} \Gamma\left(\frac{1}{3}\right)^{6}}{1024 \pi^{6}\left(\tau^{1}\right)^{3} \tau^{2} \tau^{3}} & \frac{27 \sqrt{3}|\chi|^{2} \Gamma\left(\frac{1}{3}\right)^{6}}{2048 \pi^{6}\left(\tau^{1}\right)^{2}\left(\tau^{2}\right)^{2} \tau^{3}} & \frac{27 \sqrt{3}|\chi|^{2} \Gamma\left(\frac{1}{3}\right)^{6}}{2048 \pi^{6}\left(\tau^{1}\right)^{2} \tau^{2}\left(\tau^{3}\right)^{2}} \\
\frac{27 \sqrt{3}|\chi|^{2} \Gamma\left(\frac{1}{3}\right)^{6}}{2048 \pi^{6}\left(\tau^{1}\right)^{2}\left(\tau^{2}\right)^{2} \tau^{3}} & \frac{1}{4\left(\tau^{2}\right)^{2}}+\frac{27 \sqrt{3}|\chi|^{2} \Gamma\left(\frac{1}{3}\right)^{6}}{1024 \pi^{6} \tau^{1}\left(\tau^{3}\right)^{3} \tau^{3}} & \frac{27 \sqrt{3}|\chi|^{2} \Gamma\left(\frac{1}{3}\right)^{6}}{2048 \pi^{6} \tau^{1}\left(\tau^{2}\right)^{2}\left(\tau^{3}\right)^{2}} \\
\frac{27 \sqrt{3}|\chi|^{2} \Gamma\left(\frac{1}{3}\right)^{6}}{2048 \pi^{6}\left(\tau^{1}\right)^{2} \tau^{2}\left(\tau^{3}\right)^{2}} & \frac{27 \sqrt{3}|\chi|^{2} \Gamma\left(\frac{1}{3}\right)^{6}}{2048 \pi^{6} \tau^{1}\left(\tau^{2}\right)^{2}\left(\tau^{3}\right)^{2}} & \frac{1}{4\left(\tau^{3}\right)^{2}}+\frac{27 \sqrt{3}|\chi|^{2} \Gamma\left(\frac{1}{3}\right)^{6}}{1024 \pi^{6} \tau^{1} \tau^{2}\left(\tau^{3}\right)^{3}}
\end{array}\right) \quad(i, j=1,2,3),
$$


with $\tau^{i} \equiv \operatorname{Im}\left(U^{i}\right)$, where the value of blow-up mode $|\chi|$ encodes the amount of kinetic mixing associated to a non-diagonal Kähler metric. In the blow-down limit $|\chi| \rightarrow 0$, the off-diagonal components of the Kähler metric go to zero, and these play an important role of realizing the rank-three physical Yukawa matrix with the hierarchical structure as shown below.

We numerically analyze the structure of physical Yukawa couplings $Y_{\hat{1} \hat{i} \hat{j}}, Y_{\hat{2} \hat{i} \hat{j}}$ and $Y_{\hat{3} \hat{i} \hat{j}}$ by setting

$$
\operatorname{Re}\left(U^{1}\right)=\operatorname{Re}\left(U^{2}\right)=\operatorname{Re}\left(U^{3}\right)=0, \quad \tau^{1}=3,
$$

and the other moduli values $\left(\tau^{2}, \tau^{3}, \chi\right)$ are chosen as free parameters. It turns out that the rank of physical Yukawa couplings $\left\{Y_{\hat{1} \hat{i} \hat{j}}, Y_{\hat{2} \hat{i} \hat{j}}, Y_{\hat{\mathrm{i}} \hat{i} \hat{j}}\right\}$ is three in the moduli space except for $\tau^{i}=\tau^{j}$ with $i \neq j$. When at least two moduli values are coincides with each other, two eigenvalues of the Kähler metric are degenerate due to the symmetric structure of Kähler metric with respect to $\tau^{i}$. The phenomenon is observed in the following numerical results. In a general moduli space, eigenvalues of physical Yukawa couplings $\left(m_{1}, m_{2}, m_{3}\right)$ with $\left|m_{1}\right| \geq\left|m_{2}\right| \geq\left|m_{3}\right|$ satisfy the relation

$$
\left|m_{2}\right| \simeq\left|m_{1}\right|
$$

for all $\left\{Y_{\hat{1} \hat{i} \hat{j}}, Y_{\hat{2} \hat{i} \hat{j}}, Y_{\hat{i} \hat{i} \hat{j}}\right\}$. Since we focus on the moduli space within (3.31), the off-diagonal entries in Kähler metric (3.34) should be smaller than the diagonal one. It results in two degenerate eigenvalues of physical Yukawa couplings and those are determined by the rank-two holomorphic Yukawa matrix (2.16). The smallest eigenvalue of physical Yukawa coupling has an origin in the smallness of the off-diagonal entries in the Kähler metric. Indeed, the ratio $r_{2}=\left|m_{3}\right| /\left|m_{1}\right|$ is sensitive to the value of the blow-up modulus $\chi$ as indicated in figure 6 . We plot the ratio $r_{2}$ of the physical Yukawa coupling $Y_{\hat{3} \hat{i} \hat{j}}$, but the same structure arises in the other physical Yukawa couplings.

It is remarkable that there exist a huge hierarchy between $m_{3}$ and others, due to the suppressed off-diagonal entries in Kähler metric (3.34). Indeed, the ratio of off-diagonal components to diagonal one in $|\chi| \rightarrow 0$ limit is typically of $\mathcal{O}\left(3.5 \times 10^{-2}|\chi|^{2}\left(\tau^{i}\right)^{-3}\right)$, when the moduli values $\tau^{i}$ are assumed to be the same order. From the formula of physical Yukawa couplings (2.12), the smallest ratio of eigenvalues of the physical Yukawa couplings, $r_{2}$, is then further suppressed by the value of blow-up mode through the matter Kähler metric. That phenomenon is consistent with our numerical results, indicating that the ratio $r_{2}$ becomes smaller and smaller as $|\chi|$ decreases.

\section{Conclusions}

The non-trivial structure of physical Yukawa couplings has an origin in the matter field Kähler metric and the holomorphic Yukawa couplings in the context of $4 \mathrm{D} \mathcal{N}=1$ supersymmetric theory. In the context of heterotic orbifold models, it was known that hierarchical Yukawa couplings are realized in the twisted sector due to the exponentially-suppressed holomorphic Yukawa couplings, whereas the Yukawa couplings among the untwisted modes are typically of $\mathcal{O}(1)$ in the heterotic string theory with standard embedding, where matter 

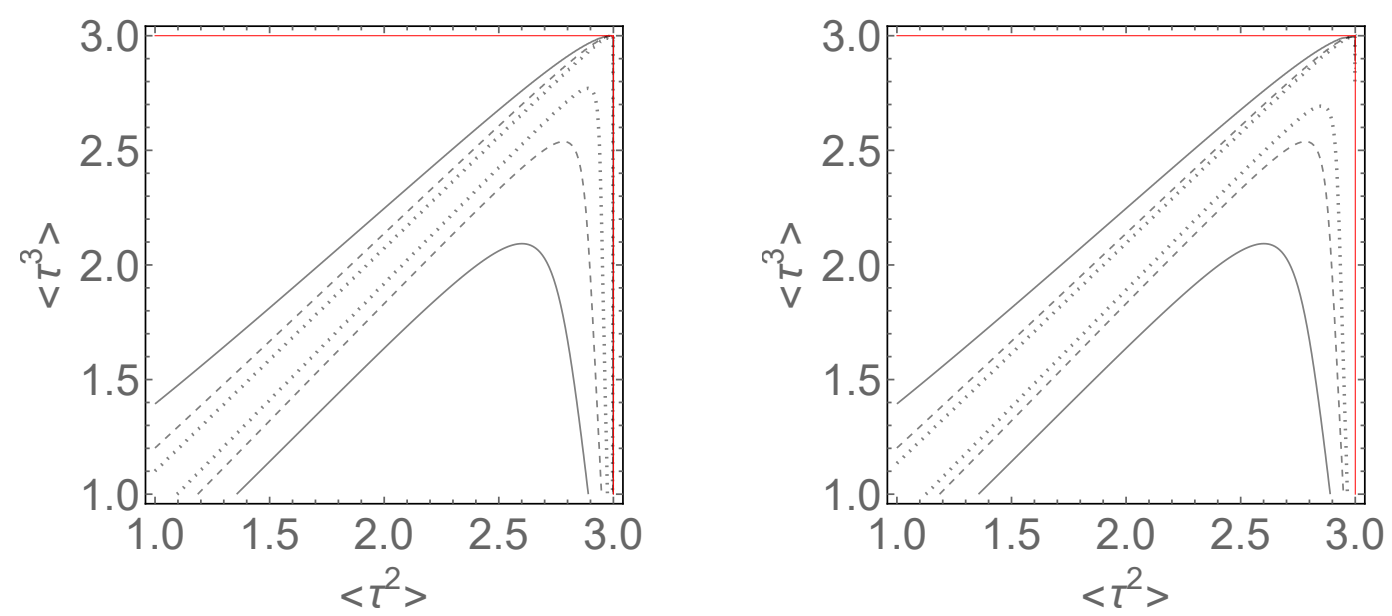

Figure 6. We plot the smallest ratio $r_{2}$ of eigenvalues of the physical Yukawa couplings $Y_{\hat{3} \hat{a} \hat{b}}$ to the maximum one with respect to $\left\langle\tau^{2}\right\rangle$ and $\left\langle\tau^{3}\right\rangle$ by setting $|\chi|=0.1$ and $|\chi|=0.01$ in the left and right panels, respectively and other moduli values are set as in eq. (3.35). In both panels, red lines correspond to $r_{2}=0$, namely rank-two Yukawa matrix. In the left (right) panel, the dotted, dashed and solid curves correspond to the values for $4 \times 10^{-5}\left(3 \times 10^{-7}\right), 2 \times 10^{-5}\left(2 \times 10^{-7}\right)$, and $10^{-5}\left(10^{-7}\right)$, respectively.

fields are assigned to the fundamental or anti-fundamental representations of $E_{6}$ gauge group. Hence, we have mainly focused on the Yukawa couplings of untwisted modes whether the matter field Kähler metric provide their hierarchical structure. Since the matter fields 27 and $\overline{27}$ are respectively in one-to-one correspondence with the Kähler moduli and complex structure moduli, one can argue definite properties of the matter field Kähler metric in the physical Yukawa couplings.

We have analyzed $27^{3}$ and $\overline{27}^{3}$ Yukawa couplings of untwisted modes on a simplified CY threefold in section 2.2.1 and the untwisted and twisted modes on toroidal orbifolds preserving the supersymmetry in section 2.2.2. It turned out that off-diagonal entries in matter field Kähler metric are necessary to generate the rank-full physical Yukawa couplings with hierarchical structure. There exists the non-vanishing matter Kähler mixing on unfactorizable tori such as $T^{6} / \mathbb{Z}_{3,4,6}$ orbifolds and blown-up toroidal orbifolds. Indeed, we performed the numerical search on $T^{6} / \mathbb{Z}_{3}$ orbifold, blown-up $T^{6} /\left(\mathbb{Z}_{2} \times \mathbb{Z}_{2}\right)$ orbifold, and a mirror dual of $T^{6} /\left(\mathbb{Z}_{3} \times \mathbb{Z}_{3}\right)$ orbifold. In the former two examples, we exhibited that the hierarchical structure of physical Yukawa couplings of matter zero-modes associated with Kähler moduli is provided by $\mathcal{O}(1)$ values of moduli fields, taking into account the realistic value of $4 \mathrm{D}$ gauge couplings. In the latter example, the complex structure moduli space accompanying chiral matter $\overline{27}$ was analyzed in detail. We found that the small but non-zero value of the blow-up mode, which is required to justify the effective action, leads to the suppressed off-diagonal components of the matter field Kähler metric, thereby providing the hierarchical structure of physical $\overline{27}^{3}$ Yukawa couplings of untwisted modes.

So far, we have focused on the realization of hierarchical structure of physical Yukawa couplings of untwisted modes by means of the structure of matter Kähler metric. These scenarios would be widely applicable to more realistic models such as $\mathrm{SU}(5)$ or $\mathrm{SO}(10)$ 
grand unified theories achieved by introducing Wilson-lines on the Cartan directions of $E_{6}$ and theories with smaller gauge groups. For example, many realistic models have been constructed in $T^{6} / \mathbb{Z}_{6-I I}$ orbifold models [41-43]. In these models, the third generation of left-handed (right-handed) quarks and leptons corresponds to the untwisted sector $A^{1}\left(A^{2}\right)$ and the Higgs field corresponds to the untwisted mode $A^{3}$, while the other two generations of quarks and leptons are originated from twisted sectors. At the orbifold limit, the Yukawa matrices are rank-one, and only the third generation can gain masses [44]. Thus, it would be interesting to extend our analysis to above models on blown-up $T^{6} / \mathbb{Z}_{6-I I}$ orbifold. $^{9}$ Including these topics, we leave a pursue of more phenomenologically interesting threegeneration models for future work.

The moduli vacuum expectation values are assumed to be free parameters in our analysis to find the structure of physical Yukawa couplings. However, a realization of the hierarchical structure of physical Yukawa couplings is highly dependent on the stabilization mechanism of the moduli fields. The moduli stabilization in the context of heterotic string theory is still open challenging issue, since it generically induces the backreaction on the background geometry. We comment on the possible mechanism of the moduli stabilization which we leave for future work. When the matter zero-modes have an origin in the Kähler moduli sector, we require some non-perturbative effects to stabilize the Kähler moduli. We often employ the gaugino condensation on the hidden sector with one-loop threshold corrections depending on the Kähler moduli and/or world-sheet instanton effects on the two-cycles of CY threefolds. When the matter zero-modes have an origin in the complex structure moduli sector, a flux compactification is a powerful tool to determine the vacuum expectation value of these moduli fields. Indeed, the flux compactification on a mirror dual of rigid CY threefolds in the example of section 3.3 is suffice to stabilize all the moduli fields. We hope to come back to these problems in the future.

\section{Acknowledgments}

T. K. was supported in part by MEXT KAKENHI Grant Number JP19H04605. H. O. was supported in part by JSPS KAKENHI Grant Numbers JP19J00664 and JP20K14477.

Open Access. This article is distributed under the terms of the Creative Commons Attribution License (CC-BY 4.0), which permits any use, distribution and reproduction in any medium, provided the original author(s) and source are credited.

\section{References}

[1] N. Arkani-Hamed and M. Schmaltz, Hierarchies without symmetries from extra dimensions, Phys. Rev. D 61 (2000) 033005 [hep-ph/9903417] [INSPIRE].

[2] D.E. Kaplan and T.M.P. Tait, Supersymmetry breaking, fermion masses and a small extra dimension, JHEP 06 (2000) 020 [hep-ph/0004200] [INSPIRE].

\footnotetext{
${ }^{9}$ See for an approach of blown-up $T^{6} / \mathbb{Z}_{6-I I}$ orbifold, ref. [45].
} 
[3] C.D. Froggatt and H.B. Nielsen, Hierarchy of quark masses, Cabibbo angles and CP-violation, Nucl. Phys. B 147 (1979) 277 [inSPIRE].

[4] H. Ishimori, T. Kobayashi, H. Ohki, Y. Shimizu, H. Okada and M. Tanimoto, Non-Abelian discrete symmetries in particle physics, Prog. Theor. Phys. Suppl. 183 (2010) 1 [arXiv: 1003.3552] [INSPIRE].

[5] H. Ishimori, T. Kobayashi, H. Ohki, H. Okada, Y. Shimizu and M. Tanimoto, An introduction to non-Abelian discrete symmetries for particle physicists, Lect. Notes Phys. $\mathbf{8 5 8}$ (2012) 1 [INSPIRE].

[6] S. Hamidi and C. Vafa, Interactions on orbifolds, Nucl. Phys. B 279 (1987) 465 [InSPIRE].

[7] L.J. Dixon, D. Friedan, E.J. Martinec and S.H. Shenker, The conformal field theory of orbifolds, Nucl. Phys. B 282 (1987) 13 [INSPIRE].

[8] T.T. Burwick, R.K. Kaiser and H.F. Muller, General Yukawa couplings of strings on $Z_{N}$ orbifolds, Nucl. Phys. B 355 (1991) 689 [InSPIRE].

[9] T. Kobayashi and N. Ohtsubo, Geometrical aspects of $Z_{N}$ orbifold phenomenology, Int. J. Mod. Phys. A 9 (1994) 87 [InSPIRE].

[10] J.A. Casas, F. Gómez and C. Muñoz, Complete structure of $Z_{N}$ Yukawa couplings, Int. J. Mod. Phys. A 8 (1993) 455 [hep-th/9110060] [INSPIRE].

[11] P. Ko, T. Kobayashi and J.-H. Park, Quark masses and mixing angles in heterotic orbifold models, Phys. Lett. B 598 (2004) 263 [hep-ph/0406041] [INSPIRE].

[12] P. Ko, T. Kobayashi and J.-H. Park, Lepton masses and mixing angles from heterotic orbifold models, Phys. Rev. D 71 (2005) 095010 [hep-ph/0503029] [INSPIRE].

[13] D. Cremades, L.E. Ibáñez and F. Marchesano, Computing Yukawa couplings from magnetized extra dimensions, JHEP 05 (2004) 079 [hep-th/0404229] [INSPIRE].

[14] H. Abe, T. Kobayashi and H. Ohki, Magnetized orbifold models, JHEP 09 (2008) 043 [arXiv:0806.4748] [INSPIRE].

[15] T. Kobayashi, H. Otsuka and H. Uchida, Wavefunctions and Yukawa couplings on resolutions of $T^{2} / Z_{N}$ orbifolds, JHEP 08 (2019) 046 [arXiv: 1904.02867] [INSPIRE].

[16] H. Abe, A. Oikawa and H. Otsuka, Wavefunctions on magnetized branes in the conifold, JHEP 07 (2016) 054 [arXiv: 1510. 03407] [INSPIRE].

[17] c. Blesneag, E.I. Buchbinder, A. Constantin, A. Lukas and E. Palti, Matter field Kähler metric in heterotic string theory from localisation, JHEP 04 (2018) 139 [arXiv: 1801.09645] [INSPIRE].

[18] L.J. Dixon, V. Kaplunovsky and J. Louis, On effective field theories describing $(2,2)$ vacua of the heterotic string, Nucl. Phys. B 329 (1990) 27 [INSPIRE].

[19] M.B. Green, J.H. Schwarz and E. Witten, Superstring theory. Volume 2: loop amplitudes, anomalies and phenomenology, Cambridge University Press, Cambridge, U.K. (1988).

[20] J. Polchinski, String theory. Volume 2: superstring theory and beyond, Cambridge University Press, Cambridge, U.K. (2007).

[21] A. Strominger and E. Witten, New manifolds for superstring compactification, Commun. Math. Phys. 101 (1985) 341 [INSPIRE].

[22] P. Candelas, Yukawa couplings between $(2,1)$ forms, Nucl. Phys. B 298 (1988) 458 [InSPIRE]. 
[23] M. Kreuzer and H. Skarke, Complete classification of reflexive polyhedra in four-dimensions, Adv. Theor. Math. Phys. 4 (2002) 1209 [hep-th/0002240] [INSPIRE].

[24] M. Kreuzer and H. Skarke, Calabi-Yau data webpage, http://hep.itp.tuwien.ac.at/ kreuzer/CY/.

[25] P. Candelas and R. Davies, New Calabi-Yau manifolds with small Hodge numbers, Fortsch. Phys. 58 (2010) 383 [arXiv:0809.4681] [InSPIRE].

[26] B.R. Greene, K.H. Kirklin, P.J. Miron and G.G. Ross, A three generation superstring model. 1. Compactification and discrete symmetries, Nucl. Phys. B 278 (1986) 667 [INSPIRE].

[27] B.R. Greene, K.H. Kirklin, P.J. Miron and G.G. Ross, A three generation superstring model. 2. Symmetry breaking and the low-energy theory, Nucl. Phys. B 292 (1987) 606 [InSPIRE].

[28] L.J. Dixon, J.A. Harvey, C. Vafa and E. Witten, Strings on orbifolds. 2, Nucl. Phys. B 274 (1986) 285 [INSPIRE].

[29] L.E. Ibáñez, J. Mas, H.-P. Nilles and F. Quevedo, Heterotic strings in symmetric and asymmetric orbifold backgrounds, Nucl. Phys. B 301 (1988) 157 [INSPIRE].

[30] A. Font, L.E. Ibáñez and F. Quevedo, $Z_{N} \times Z_{M}$ orbifolds and discrete torsion, Phys. Lett. $B$ 217 (1989) 272 [INSPIRE].

[31] Y. Katsuki, Y. Kawamura, T. Kobayashi, N. Ohtsubo, Y. Ono and K. Tanioka, $Z_{N}$ orbifold models, Nucl. Phys. B 341 (1990) 611 [inSPIRE].

[32] M. Fischer, S. Ramos-Sanchez and P.K.S. Vaudrevange, Heterotic non-Abelian orbifolds, JHEP 07 (2013) 080 [arXiv: 1304.7742] [INSPIRE].

[33] S. Ferrara, C. Kounnas and M. Porrati, General dimensional reduction of ten-dimensional supergravity and superstring, Phys. Lett. B 181 (1986) 263 [INSPIRE].

[34] M. Cvetič, J. Louis and B.A. Ovrut, A string calculation of the Kähler potentials for moduli of $Z_{N}$ orbifolds, Phys. Lett. B 206 (1988) 227 [INSPIRE].

[35] L.E. Ibáñez and D. Lüst, Duality anomaly cancellation, minimal string unification and the effective low-energy Lagrangian of $4 D$ strings, Nucl. Phys. B 382 (1992) 305 [hep-th/9202046] [INSPIRE].

[36] H. Abe, T. Kobayashi, H. Otsuka, Y. Takano and T.H. Tatsuishi, Gauge coupling unification in $\mathrm{SO}(32)$ heterotic string theory with magnetic fluxes, PTEP 2016 (2016) 053B01 [arXiv: 1507.04127] [INSPIRE].

[37] M. Cvetič, B.A. Ovrut and J. Louis, The Zamolodchikov metric and effective Lagrangians in string theory, Phys. Rev. D 40 (1989) 684 [InSPIRE].

[38] F. Denef, M.R. Douglas, B. Florea, A. Grassi and S. Kachru, Fixing all moduli in a simple F-theory compactification, Adv. Theor. Math. Phys. 9 (2005) 861 [hep-th/0503124] [INSPIRE].

[39] M. Blaszczyk, S. Groot Nibbelink, F. Ruehle, M. Trapletti and P.K.S. Vaudrevange, Heterotic MSSM on a resolved orbifold, JHEP 09 (2010) 065 [arXiv: 1007.0203] [inSPIRE].

[40] P. Candelas, E. Derrick and L. Parkes, Generalized Calabi-Yau manifolds and the mirror of a rigid manifold, Nucl. Phys. B 407 (1993) 115 [hep-th/9304045] [INSPIRE].

[41] T. Kobayashi, S. Raby and R.-J. Zhang, Searching for realistic 4d string models with a Pati-Salam symmetry: orbifold grand unified theories from heterotic string compactification on a $Z_{6}$ orbifold, Nucl. Phys. B 704 (2005) 3 [hep-ph/0409098] [INSPIRE]. 
[42] W. Buchmüller, K. Hamaguchi, O. Lebedev and M. Ratz, Supersymmetric Standard Model from the heterotic string (II), Nucl. Phys. B 785 (2007) 149 [hep-th/0606187] [INSPIRE].

[43] O. Lebedev et al., A mini-landscape of exact MSSM spectra in heterotic orbifolds, Phys. Lett. $B 645$ (2007) 88 [hep-th/0611095] [INSPIRE].

[44] P. Ko, T. Kobayashi, J.-H. Park and S. Raby, String-derived $D_{4}$ flavor symmetry and phenomenological implications, Phys. Rev. D 76 (2007) 035005 [Erratum ibid. 76 (2007) 059901] [arXiv: 0704.2807] [INSPIRE].

[45] S. Groot Nibbelink, J. Held, F. Ruehle, M. Trapletti and P.K.S. Vaudrevange, Heterotic $Z_{6-I I}$ MSSM orbifolds in blowup, JHEP 03 (2009) 005 [arXiv: 0901.3059] [INSPIRE]. 